\title{
Dynamics of a discontinuous coupled electro-mechanical system oscillator with strong irrational nonlinearities and with two outputs
}

\author{
Danao Adile Adoum 1, Ali Ramadan 1, Samuel Noubissié 2, Mahmat Tahir Abakar ${ }^{1}$, Hervé Simo 3, Fabien \\ Kenmogne ${ }^{4,}{ }^{*}$, Timothée Thierry Odi Enyegue ${ }^{4}$, and Malloum Soultan ${ }^{5}$ \\ ${ }^{1}$ Department of industrial engineering and maintenance, polytechnic university of Mongo, Chad \\ ${ }^{2}$ Research unit of industrial systems engineering and environment (RU-ISEE), Fotso Victor University Institute of \\ Technology, University of Dschang, Cameroon. \\ ${ }^{3}$ Department of Mechanical Engineering, the University Institute of Technology, P.O. Box 455, The University of \\ Ngaoundéré, Cameroon \\ ${ }^{4}$ Department of Civil Engineering, Higher Teacher Training College of the Technical Education, P.O. Box 1872, University \\ of Douala, Cameroon \\ ${ }^{5}$ Department of technical sciences, faculty of exact and applied sciences, P.O. Box 4377 University of Djamena, Chad
}

Global Journal of Engineering and Technology Advances, 2021, 06(01), 116-135

Publication history: Received on 17 January 2021; revised on 24 January 2021; accepted on 26 January 2021

Article DOI: https://doi.org/10.30574/gjeta.2021.6.1.0301

\begin{abstract}
The dynamics of the nonlinear electromechanical device, consisting of a mechanical part with two outputs and an electrical part which acts as the server is strongly investigated in the present work. The mechanical part consists of two nonlinear oscillators with strong irrational nonlinearities having smooth or discontinuous characteristics, where nonlinearity is just due to the inclination of springs, the geometric configuration, which are both elastically coupled. While the electrical part is the $\phi^{6}$ Rayleigh equation. By using the Lagrangian formulation, the model equations are established and used to investigate the equilibrium points and their stabilities. Nest by using the multiple time scales method, the analytical solutions are found both for the case of large amplitude and the weak amplitude, leading to interesting bifurcation sets of the equilibria by varying the control parameters, the inclination angles and driven frequency. Finally, numerical investigations of the exact equation of the system are used to justify the validity of analytical results and to find new phenomena such as chaotic impulses, chaotic bursting and the train of kink signal generations.
\end{abstract}

Keywords: Irrational Nonlinearity; Electromechanical Device; Chaotic Impulse; Chaotic Bursting.

\section{Introduction}

Nowadays, much more attentions are focused on nonlinear systems useful in the design and modeling of some practical systems, and this in most scientific and technology applications [1, 2, 3]. To name just a few, Qingjie Cao et al. proposed an archetypal system to investigate transitions from smooth to discontinuous dynamics, which in the smooth regime, bears significant similarities to the Duffing oscillator, exhibiting the standard dynamics governed by the hyperbolic structure associated with the stationary state of the double well, while Dupac et al. [5] modeled an electromagnetically levitated droplet as a three-dimensional system with lumped masses and elastic springs where its nonlinear behaviour was investigated. Next Boubaker et al. [6] investigated the mesoscopic fabric models by employing a discrete massspring approach. Terumichi et al. [7] proposed a mass-spring system attached at the lower end to a time-varying length

\footnotetext{
${ }^{*}$ Corresponding author: Fabien Kenmogne; Email: cabenset@yahoo.fr

Department of Civil Engineering, Higher Teacher Training College of the Technical Education,P.O. Box 1872, University of Douala, Cameroon.
} 
string and the non-stationary vibrations were investigated. Although certain single autonomous oscillators like the Shua, Colpits and Vander Pol have been proved to have riches dynamics, the driven versions of these oscillators have richer dynamics and this being due to the fact that the time varying excitation introduces one more degree of freedom in the dynamical systems [8]. Let us mention that some of the driven oscillators like the Duffing one have oscillations which vanish when the driven force is removed. In addition, it has been proved that when these oscillators are coupled each to others, the resulting system will have more applications, among which the increasing of the frequency range of signals generated, the increasing of the generated powers as well as the conversion of one form of energy to other, with different dynamics [9]. For e.g. the periodic regular electrical signal can be converted to chaotic mechanical motion and vice versa.

Nature is full of nonlinearities, responsible for a great variety of responses in natural systems. Natural behaviors could either be periodic or chaotic over time and space. Let us mention that all technology innovations are consequences of the development in scientific tools, and this after strong mathematical investigations. New phenomena in nonlinear dynamics were discovered because of chaotic and unpredictable behavior from apparently deterministic systems, including mechanical systems. It is known that the chaotic motion of dynamical systems cannot always be predicted far into the future. Generally, dynamical systems depend on one or several parameters that control the strength of external influences. For example in forced oscillations, the frequency and amplitude of the exciting oscillation are the control parameters. In varying these parameters one may hit critical values at which the behaviors of systems change qualitatively. Critical values of this kind are called bifurcations. Bifurcations play an important role in the development of deterministic chaos. Many mechanical systems, being them single or coupled can be used to see the effects of nonlinear dynamics.

The attention carried on researches in the area of coupled non-linear oscillators has received a great particular attention in recent years $[10,11]$. This being due to the fact that coupled oscillators provide fundamental models for the dynamics of various physical, electrical, mechanical and biological systems $[12,13,14]$. The electro-mechanical systems, that is systems with coupled mechanical structures and electrical/electromagnetic circuits, are widely used in mechatronics for a variety of applications (e.g. as actuators, sensors, servo mechanisms, switches,...). Dynamics of such systems have been proved to be possible by means of two mutually coupled differential equations that are the equations describing both the electrical, and the mechanical parts.

In [4], Y W Han, et al. proposed a nonlinear oscillator with a pair of irrational nonlinearities, which lead to the transition from smooth to discontinuous dynamics, this nonlinear oscillator being comprised a lumped mass, linked by a pair of inclined elastic linear springs. As a consequence the nonlinearity obtained was strong and due just to the inclination. In this work, we focus on the elastically coupling of two of the previous models of nonlinear oscillators, both magnetically coupled to nonlinear electrical Rayleigh circuit. As we shall see further, the obtained circuit has richer dynamics and would provide a variety of applications in engineering.

\section{Description of the system model and equations of motion}

We consider the dynamic response of an electromechanical instrument consisting of an electrical part described by the $\phi^{6}$ Rayleigh equation [8] coupled to the mechanical part as depicted in Fig.1. The mechanical part consists of two simple mechanical systems, elastically coupled through the spring with stiffness $k_{s}$. Each mechanical system comprises a lumped mass, $m_{i}(i=1,2)$, linked by a pair of inclined elastic springs of stiffness $k_{i}(i=1,2)$, capable of resisting both tension and compression and which are pinned to their rigid supports (T). Although each of the inclined springs provides linear restoring resistance, it has been proved that the resulting force has a strong irrational nonlinearity due to the geometric configuration [4]. The electrical part consists of a linear inductor L, a nonlinear capacitor (NC), a nonlinear resistor (NR) in series with electromotive force $\mathrm{e}(\mathrm{t})$. The voltage over the NR and the NC can be expressed as $V_{N R}=-R\left(1-\frac{\dot{q}^{2}}{\omega_{0}^{2} Q_{0}^{2}}\right) \dot{q}$ and $V_{N C}=\frac{q}{c_{0}}\left(1-\frac{\alpha_{\mathrm{a}}}{\mathrm{Q}_{0}^{2}} \mathrm{q}^{2}+\frac{\alpha_{\mathrm{b}} \mathrm{q}^{4}}{\mathrm{Q}_{0}^{4}}\right)$, where by the dot, we mean the derivative with respect to time t. $\alpha_{a}$ being a dimensionless parameter which can be positive or negative, while $\alpha_{b}$ is a positive dimensionless parameter. These parameters account of nonlinear diode used. $\omega_{0}$ and $Q_{0}$ are the nominal angular frequency and charge, respectively. 


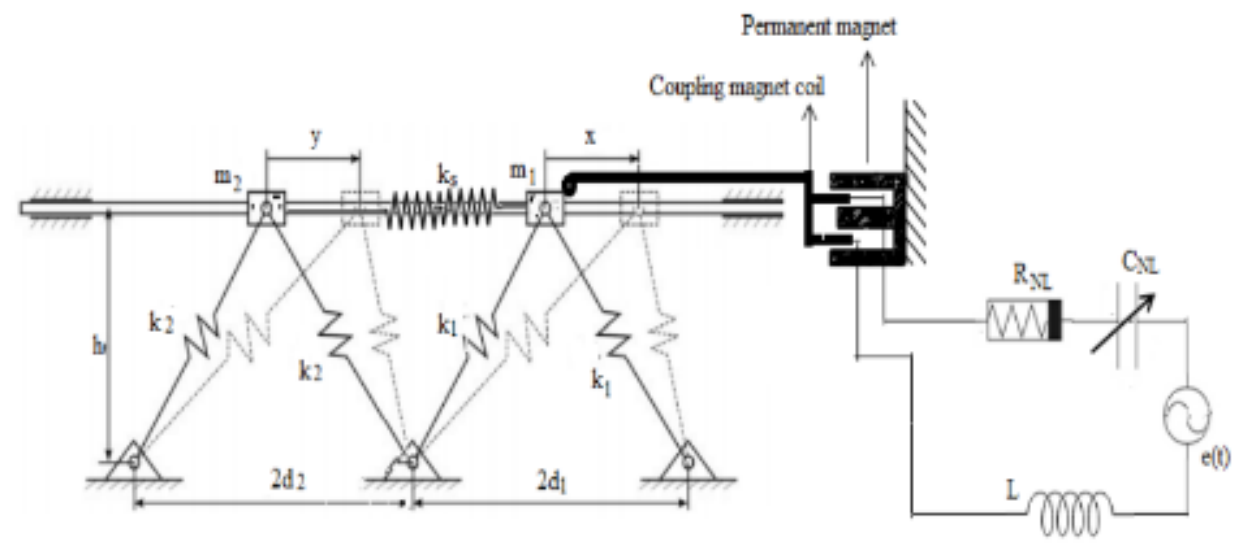

Figure 1 Circuit diagram of the electromechanical instrument, driven by the low frequency signal generator.

The coupling between the electrical and mechanical parts is made by the electromagnetic transducer where the first coupling equation follows from Faraday's law: A coil of $n$ turns moving at the velocity $\mathrm{v}$ with respect to the magnetic flux density B generates an electromotive force (voltage) given by $e=2 \pi n r B v=B l \dot{x}$. The second equation follows from the Lorentz force law: The external force f required to balance the total force of the magnetic field on turns of the conductor is $f=-2 \pi n r B i=-B l \dot{q}$. The kinetic energy of the system is then deduced as:

$T=\frac{1}{2} m_{1} \dot{x}^{2}+\frac{1}{2} m_{2} \dot{y}^{2}+\frac{1}{2} L \dot{q}^{2}+\frac{1}{2} B l(x \dot{q}-q \dot{x})$

where the over dot is the derivative with respect to time $\mathrm{t}$ and where $\mathrm{x}$ and $\mathrm{y}$ are the displacements of masses $m_{1}$ and $m_{2}$, respectively. The potential energy is:

$V=+\frac{1}{2} k_{1}\left[\left(\sqrt{\left(d_{1}+x\right)^{2}+h^{2}}-\sqrt{d_{1}^{2}+h^{2}}\right)^{2}+\left(\sqrt{\left(d_{1}-x\right)^{2}+h^{2}}-\sqrt{d_{1}^{2}+h^{2}}\right)^{2}\right]+\frac{1}{2} k_{2}\left[\left(\sqrt{\left(d_{2}+y\right)^{2}+h^{2}}-\right.\right.$
$\left.\left.\sqrt{d_{2}^{2}+h^{2}}\right)^{2}+\left(\sqrt{\left(d_{2}-y\right)^{2}+h^{2}}-\sqrt{d_{2}^{2}+h^{2}}\right)^{2}\right]+\frac{1}{2} k_{s}(y-x)^{2}+\frac{1}{2 c_{0}} q^{2}-\frac{\alpha_{a}}{4 Q_{0}^{2}} q^{4}+\frac{\alpha_{b}}{6 Q_{0}^{4}} q^{6}$.

While the dissipative function is expressed as:

$D=\frac{1}{2} \lambda_{1} \dot{x}^{2}+\frac{1}{2} \lambda_{2} \dot{y}^{2}-\frac{1}{2} R\left(1-\frac{\dot{q}^{2}}{2 \omega_{0}^{2} Q_{0}^{2}}\right) \dot{q}^{2}$

From the above defined energy components, one can define the Lagrangian given as $L=T-V$, while the equation of motion can be obtained from the Lagrangian equation

$\frac{d}{d t}\left(\frac{\partial L}{\partial \dot{x}}\right)-\frac{\partial L}{\partial x}=-\frac{\partial D}{\partial \dot{x}}, \frac{d}{d t}\left(\frac{\partial L}{\partial \dot{y}}\right)-\frac{\partial L}{\partial y}=-\frac{\partial D}{\partial \dot{y}}, \quad \frac{d}{d t}\left(\frac{\partial L}{\partial \dot{q}}\right)-\frac{\partial L}{\partial q}=-\frac{\partial D}{\partial \dot{q}}+e(t)$

leading to the following equation of motion:

$$
\left\{\begin{array}{c}
m_{1} \ddot{x}+\lambda_{1} \dot{x}+2 k_{1} x-k_{1} \sqrt{d_{1}^{2}+h^{2}}\left(\frac{\left(x+d_{1}\right)}{\sqrt{\left(x+d_{1}\right)^{2}+h^{2}}}+\frac{\left(x-d_{1}\right)}{\sqrt{\left(x-d_{1}\right)^{2}+h^{2}}}\right)+k_{s}(x-y)-B l \dot{q}=0 \\
m_{2} \ddot{y}+\lambda_{2} \dot{y}+2 k_{2} y-k_{2} \sqrt{d_{2}^{2}+h^{2}}\left(\frac{\left(y+d_{2}\right)}{\sqrt{\left(y+d_{2}\right)^{2}+h^{2}}}+\frac{\left(y-d_{2}\right)}{\sqrt{\left(y-d_{2}\right)^{2}+h^{2}}}\right)+k_{s}(y-x)=0 \\
L \ddot{q}-R\left(1-\frac{\dot{q}^{2}}{\omega_{0}^{2} Q_{0}^{2}}\right) \dot{q}+\frac{1}{C_{0}} q-\frac{\alpha_{\mathrm{a}}}{\mathrm{Q}_{0}^{2}} q^{3}+\frac{\alpha_{\mathrm{b}}}{\mathrm{Q}_{0}^{4}} q^{5}+B l \dot{x}=e_{0} \cos (\omega t) .
\end{array}\right.
$$

Let us make the changes of variables and parameters:

$X=\frac{x}{\sqrt{d_{1}^{2}+h^{2}}}, Y=\frac{y}{\sqrt{d_{2}^{2}+h^{2}}}, Z=\frac{q}{Q_{0}}, \tau=t \omega_{e}, \omega_{e}=\frac{1}{\sqrt{L C_{0}}}$,

leading to the following dimensionless equation: 


$$
\begin{aligned}
& \left\{\begin{array}{c}
\ddot{X}+\sigma_{1} \dot{X}+2 \omega_{1}^{2} X-\omega_{1}^{2}\left(\frac{X+\cos \left(\theta_{1}\right)}{\sqrt{\left(X+\cos \left(\theta_{1}\right)\right)^{2}+\sin ^{2}\left(\theta_{1}\right)}}+\frac{X-\cos \left(\theta_{1}\right)}{\sqrt{\left(X-\cos \left(\theta_{1}\right)\right)^{2}+\sin ^{2}\left(\theta_{1}\right)}}\right)+\gamma_{1}\left(X-\varepsilon_{1} Y\right)-\beta_{1} \dot{Z}=0, \\
\ddot{Y}+\sigma_{2} \dot{Y}+2 \omega_{2}^{2} Y-\omega_{2}^{2}\left(\frac{Y+\cos \left(\theta_{2}\right)}{\sqrt{\left(Y+\cos \left(\theta_{2}\right)\right)^{2}+\sin ^{2}\left(\theta_{2}\right)}}+\frac{Y-\cos \left(\theta_{2}\right)}{\sqrt{\left(Y-\cos \left(\theta_{2}\right)\right)^{2}+\sin ^{2}\left(\theta_{2}\right)}}\right)-\gamma_{2}\left(X-\varepsilon_{1} Y\right)=0, \\
\ddot{Z}-\sigma_{3}\left(1-\alpha_{0} \dot{Z}^{2}\right) \dot{Z}+Z-\alpha_{1} Z^{3}+\alpha_{2} Z^{5}+\beta_{2} \dot{X}=E_{0} \cos (\Omega t),
\end{array}\right. \\
& \sigma_{1}=\frac{\lambda_{1}}{m_{1} \omega_{e}}, \omega_{1}^{2}=\frac{k_{1}}{m_{1} \omega_{e}^{2}}, \gamma_{1}=\frac{k_{s}}{m_{1} \omega_{e}^{2}}, \beta_{1}=\frac{B l Q_{0}}{m_{1} \omega_{e} \sqrt{d_{1}^{2}+h^{2}}}, \sigma_{2}=\frac{\lambda_{2}}{m_{2} \omega_{e}}, \omega_{2}^{2}=\frac{k_{2}}{m_{2} \omega_{e}^{2}}, \gamma_{2}=\frac{k_{s}}{m_{2} \varepsilon_{1} \omega_{e}^{2}}, \\
& \sigma_{3}=\frac{R}{L \omega_{e}}, \alpha_{0}=\frac{\omega_{e}^{2}}{\omega_{0}^{2}}, \alpha_{1}=C \alpha_{a}, \alpha_{2}=C \alpha_{b}, \tan \left(\theta_{1}\right)=\frac{h}{d_{1}}, \tan \left(\theta_{2}\right)=\frac{h}{d_{2}}, \\
& \beta_{2}=\frac{B l \sqrt{d_{1}^{2}+h^{2}}}{L Q_{0} \omega_{e}}, E_{0}=C e_{0}, \Omega=\frac{\omega}{\omega_{e}}, \quad \varepsilon_{1}=\frac{\sqrt{d_{2}^{2}+h^{2}}}{\sqrt{d_{1}^{2}+h^{2}}}
\end{aligned}
$$

The mechanical parts the above equations (7) have already been found in [4], in which in place of $\cos (\theta)$ and $\sin (\theta)$, they had $\alpha$ and $\beta$, respectively. With $\alpha=\frac{h}{L}$ and $\beta=\frac{d}{L^{\prime}}$, while $L=\sqrt{h^{2}+d^{2}}$. As one can see, $\alpha^{2}+\beta^{2}=1$, meaning that $\alpha$ and $\beta$ of the differential equation obtained in [4] could not be treated as independent parameters as it was the case. This is why in order to avoid this misunderstanding, we use here $\cos (\theta)$ and $\sin (\theta)$ in place of the old $\alpha$ and $\beta$ since $\cos ^{2}(\theta)+\sin ^{2}(\theta)=1$.For the large amplitude oscillations, or for weak value of angles $\theta_{1}$ and $\theta_{2}$ that is for $\mid X \pm$ $\cos \left(\theta_{1} \mid \gg \sin \left(\theta_{1}\right)\right.$ and $\left|Y \pm \cos \left(\theta_{2}\right)\right| \gg \sin \left(\theta_{2}\right)$, equation (7) leads to

$$
\left\{\begin{array}{c}
\ddot{X}+\sigma_{1} \dot{X}+2 \omega_{1}^{2} X-\omega_{1}^{2}\left(\operatorname{csgn}\left(X+\cos \left(\theta_{1}\right)\right)+\operatorname{csgn}\left(X-\cos \left(\theta_{1}\right)\right)+\gamma_{1}\left(X-\varepsilon_{1} Y\right)-\beta_{1} \dot{Z}=0,\right. \\
\ddot{Y}+\sigma_{2} \dot{Y}+2 \omega_{2}^{2} Y-\omega_{2}^{2}\left(\operatorname{csgn}\left(Y+\cos \left(\theta_{2}\right)\right)+\operatorname{csgn}\left(Y-\cos \left(\theta_{2}\right)\right)+\gamma_{2}\left(\varepsilon_{1} Y-X\right)=0,\right. \\
\ddot{Z}-\sigma_{3}\left(1-\alpha_{0} \dot{Z}^{2}\right) \dot{Z}+Z-\alpha_{1} Z^{3}+\alpha_{2} Z^{5}+\beta_{2} \dot{X}=E_{0} \cos (\Omega t),
\end{array}\right.
$$

$\operatorname{csgn}(\mathrm{x})$ being the signum function.

$$
\operatorname{csgn}(x)=\left\{\begin{array}{c}
-1 \text { if } x<0 \\
1 \text { if } x \geq 0
\end{array}\right.
$$

For weak amplitude oscillations, Eq.(7) can be expanded to sixth order to give:

$$
\left\{\begin{array}{c}
\ddot{X}+\sigma_{1} \dot{X}+2 \omega_{1}^{2} \backslash \cos ^{2}\left(\theta_{1}\right) X-\omega_{1}^{2} \sin ^{2}\left(\theta_{1}\right)\left(5 \cos ^{2}\left(\theta_{1}\right)-1\right) X^{3}- \\
\frac{3}{4} \omega_{1}^{2} \sin ^{2}\left(\theta_{1}\right)\left(21 \cos ^{4}\left(\theta_{1}\right)-14 \cos ^{2}\left(\theta_{1}\right)+1\right) X^{5}+\gamma_{1}\left(X-\varepsilon_{1} Y\right)-\beta_{1} \dot{Z}=0, \\
\ddot{Y}+\sigma_{2} \dot{Y}+2 \omega_{2}^{2} \cos ^{2}\left(\theta_{2}\right) Y-\omega_{2}^{2} \sin ^{2}\left(\theta_{2}\right)\left(5 \cos ^{2}\left(\theta_{2}\right)-1\right) Y^{3}- \\
\frac{3}{4} \omega_{2}^{2} \sin ^{2}\left(\theta_{2}\right)\left(21 \cos ^{4}\left(\theta_{2}\right)-14 \cos ^{2}\left(\theta_{2}\right)+1\right) Y^{5}+\gamma_{2}\left(\varepsilon_{1} Y-X\right)=0, \\
\ddot{Z}-\sigma_{3}\left(1-\alpha_{0} \dot{Z}^{2}\right) \dot{Z}+Z-\alpha_{1} Z^{3}+\alpha_{2} Z^{5}+\beta_{2} \dot{X}=E_{0} \cos (\Omega t),
\end{array}\right.
$$

\section{Equilibrium points and stability}

\subsection{Equilibrium points}

In order to find the equilibrium points of the system, let us rewrite the set of Eqs.(7) in the vector form as $\dot{u}=F(u)+$ $E(t)$, where $u=(X, \dot{X}, Y, \dot{Y}, Z, \dot{Z})$. The non-linear flow $\mathrm{F}$ represents the motion equations of the non-excited system and $E$ is the external excitation. The equilibrium states $u_{e q}$ are solutions of the set of non-linear algebraic equations $F\left(u_{e q}\right)=0$, that is

$$
\left\{\begin{array}{c}
2 \omega_{1}^{2} X-\omega_{1}^{2}\left(\frac{X+\cos \left(\theta_{1}\right)}{\sqrt{\left(X+\cos \left(\theta_{1}\right)\right)^{2}+\sin ^{2}\left(\theta_{1}\right)}}+\frac{X-\cos \left(\theta_{1}\right)}{\sqrt{\left(X-\cos \left(\theta_{1}\right)\right)^{2}+\sin ^{2}\left(\theta_{1}\right)}}\right)+\gamma_{1}\left(X-\varepsilon_{1} Y\right)=0, \\
2 \omega_{2}^{2} Y-\omega_{2}^{2}\left(\frac{Y+\cos \left(\theta_{2}\right)}{\sqrt{\left(Y+\cos \left(\theta_{2}\right)\right)^{2}+\sin ^{2}\left(\theta_{2}\right)}}+\frac{Y-\cos \left(\theta_{2}\right)}{\sqrt{\left(Y-\cos \left(\theta_{2}\right)\right)^{2}+\sin ^{2}\left(\theta_{2}\right)}}\right)-\gamma_{2}\left(X-\varepsilon_{1} Y\right)=0, \\
Z-\alpha_{1} Z^{3}+\alpha_{2} Z^{5}=0 .
\end{array}\right.
$$


In order to simplify our studies, let us impose $d_{1}=d_{2}=d$, leading that $\theta_{1}=\theta_{2}=\theta=\arctan (h / d)$ and $\varepsilon_{1}=1$. One can then have at the equilibrium point, $x_{0}=y_{0}=R_{0}, R_{0}$ being the solution of the following equation:

$2 R_{0}-\left(\frac{R_{0}+\cos (\theta)}{\sqrt{\left(R_{0}+\cos (\theta)\right)^{2}+\sin ^{2}(\theta)}}+\frac{R_{0}-\cos (\theta)}{\sqrt{\left(R_{0}-\cos (\theta)\right)^{2}+\sin ^{2}(\theta)}}\right)=0$.

admitting as solutions $R_{0}=0$, and/or $R_{0}=R$, where $\mathrm{R}$ is the solution of the following polynomial equation:

$$
\begin{aligned}
& 2 R^{10}-2(1+4 \cos (2 \theta)) R^{8}+(9+4 \cos (4 \theta)+7 \cos (2 \theta)) R^{6}-(6+3 \cos (4 \theta)+11 \cos (2 \theta)) R^{4}+ \\
& (4+5 \cos (2 \theta)+\cos (4 \theta)) R^{2}-2\left(\sin ^{6}(\theta)+\cos ^{2}(\theta)-\sin ^{4}(\theta)=0 .\right.
\end{aligned}
$$

The obtained solution is plotted in Fig.2, showing for each value of $\theta$ two solutions with opposite signs. Since $\alpha_{2}>0$, it appears that the third line of Eq.(12) will admit the following solution:

If $\alpha_{1}<2 \sqrt{\alpha_{2}}$, one has only one solution $Z_{0}=0$, leading the system to three possible equilibrium points $\left(X_{0}, Y_{0}, Z_{0}\right)=$ $(0,0,0)$, that is the trivial solution and $\left(X_{0}, Y_{0}, Z_{0}\right)=( \pm R, \pm R, 0)$, R being the solution of

Eq.(13) as plotted in Fig.2.

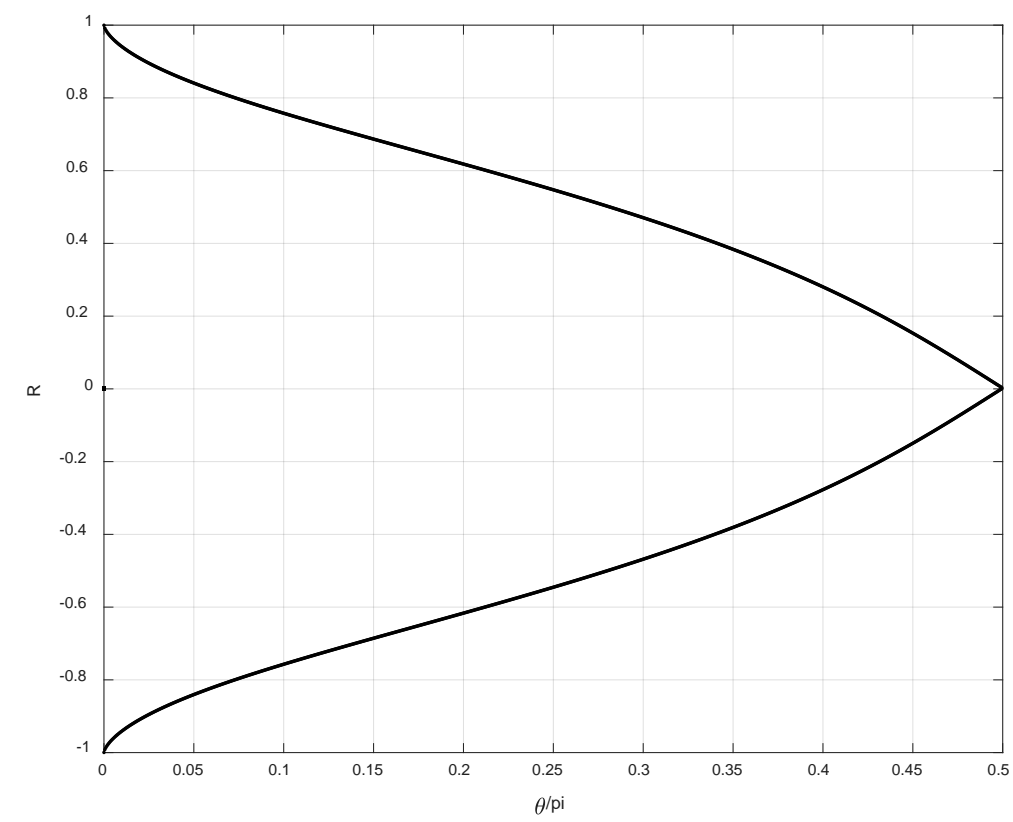

Figure 2 Evolution of equilibrium point related to Eq. (14) with respect to phase $\theta / \pi$. It is obvious that Eq.13 admits three solutions

If $\alpha_{1}=2 \sqrt{\alpha_{2}}$, the last line of Eq.(12) has as solution $Z_{0}=0$ or $Z_{0}= \pm \sqrt{\frac{\alpha_{1}}{2 \alpha_{2}}}$, meaning that the system will admit the combination of 3 fixed points for the first two lines and 3 others for the third line, which give $3 \times 3=9$ equilibrium points.

For $\alpha_{1}>2 \sqrt{\alpha_{2}}$, the last line of Eq.(12) has as solution

$$
Z_{1 \pm}= \pm \sqrt{\frac{\left(\alpha_{1}+\sqrt{\alpha_{1}^{2}-4 \alpha_{2}}\right)}{2 \alpha_{2}}}, Z_{2 \pm}= \pm \sqrt{\frac{\left(\alpha_{1}-\sqrt{\alpha_{1}^{2}-4 \alpha_{2}}\right)}{2 \alpha_{2}}}
$$

meaning that the system will admit the combination of 3 fixed points for the first two lines and 5 others for the third line, which gives $3 \times 5=15$ equilibrium points. 
For these obvious reasons the system will have the multi stability-like behaviour. To prove the above remarks, let us mention that for $\varepsilon_{1}=1$ and $\gamma_{1}=\gamma_{2}$, the set of Eq.(11) admits the potential energy

$V(X, Y, Z)=\frac{1}{2} \omega_{1}^{2}\left[\left(\sqrt{(X+\cos (\theta))^{2}+\backslash \sin ^{2}(\theta)}-1\right)^{2}+\left(\sqrt{(X-\cos (\theta))^{2}+\sin ^{2}(\theta)}-1\right)^{2}\right]+$

$\frac{1}{2} \omega_{2}^{2}\left[\left(\sqrt{(Y+\cos (\theta))^{2}+\sin ^{2}(\theta)}-1\right)^{2}+\left(\sqrt{(Y-\cos (\theta))^{2}+\sin ^{2}(\theta)}-1\right)^{2}\right]+$

$\frac{1}{2} \gamma_{1}(X-Y)^{2}+\frac{1}{2} Z^{2}-\frac{1}{4} \alpha_{1} Z^{4}+\frac{1}{6} \alpha_{2} Z^{6}$

This potential energy is plotted in Fig.(3) for the case $X=Y, \omega_{1}=\omega_{2}=\frac{1}{\sqrt{2}}, \theta=0.2, \alpha_{2}=1$. In Fig.(3) (a), we have the case $\alpha_{1}=3>2 \sqrt{2 \alpha_{2}}$, while in Fig.(3)(b) the case $\alpha_{1}=1<2 \sqrt{\alpha_{2}}$. These graphs are in agreement with the above analysis.

(a)

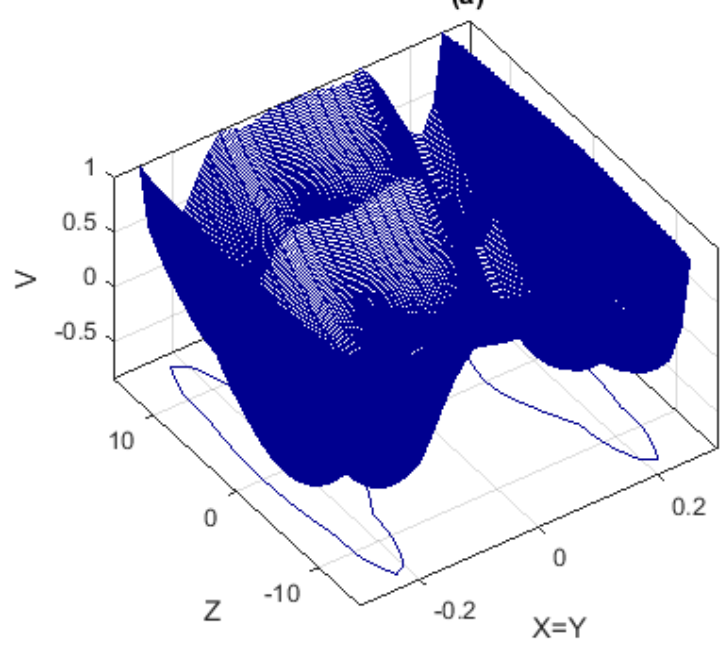

(b)

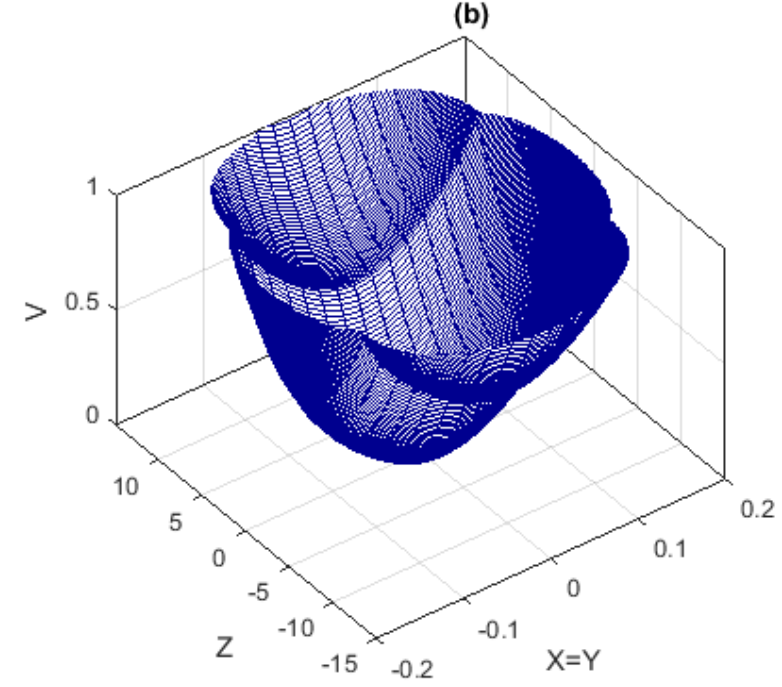

Figure 3 Potential energy according to Eq.(16) obtained for, (a): $\boldsymbol{\alpha}_{2}=1$, and $\alpha 1=3>2 \sqrt{\boldsymbol{\alpha}_{2}}$.

(b): $\alpha_{2}=1$ and $\alpha_{1}=1<\sqrt{\alpha_{2}}$.

\subsection{Stability of equilibrium points}

The local stability analysis of the above equilibrium points can be determined by investigating the linearized system $\delta \dot{u}=J \delta u$, $\mathrm{J}_{\text {is }}$ the six $^{\text {th }}$ order Jacobean matrix defined by $J=\frac{\partial F(u)}{\partial u}$, leading to:

$J=\left(\begin{array}{cccccc}0 & 1 & 0 & 0 & 0 & 0 \\ -\xi_{1} & -\sigma_{1} & \gamma_{1} & 0 & 0 & \beta_{1} \\ 0 & 0 & 0 & 1 & 0 & 0 \\ \gamma_{2} & 0 & -\xi_{2} & -\sigma_{2} & 0 & 0 \\ 0 & 0 & 0 & 0 & 0 & 1 \\ 0 & -\beta_{2} & 0 & 0 & \mathrm{j}_{2} & \sigma_{3}\end{array}\right), \xi_{1}=\omega_{1}^{2} \mathrm{j}_{1}+\gamma_{1}, \xi_{2}=\omega_{2}^{2} \mathrm{j}_{1}+\gamma_{2}$

with $j_{1}=2-\sin ^{2}(\theta)\left(\frac{1}{\left((R+\cos (\theta))^{2}+\sin ^{2}(\theta)\right)^{3 / 2}}+\frac{1}{\left((R-\cos (\theta))^{2}+\sin ^{2}(\theta)\right)^{3 / 2}}\right), j_{2}=-1+3 \alpha_{1} Z_{0}^{2}-5 \alpha_{2} Z_{0}^{4}$. This jacobean matrix leads to the characteristic equation

$$
\lambda^{6}+p_{0} \lambda^{5}+p_{1} \lambda^{4}+p_{2} \lambda^{3}+p_{3} \lambda^{2}+p_{4} \lambda+p_{5}=0
$$


With

$p_{0}=\sigma_{1}+\sigma_{2}-\sigma_{3}, p_{1}=\left(\omega_{2}^{2}+\omega_{1}^{2}\right) j_{1}+\beta_{2} \beta_{1}-j_{2}-\sigma_{3} \sigma_{2}-\sigma_{3} \sigma_{1}+\gamma_{1}+\gamma_{2}+\sigma_{2} \sigma_{1}$

$p_{2}=\left(-\sigma_{3} \omega_{1}^{2}+\sigma_{1} \omega_{2}^{2}+\sigma_{2} \omega_{1}^{2}-\sigma_{3} \omega_{2}^{2}\right) j_{1}+\beta_{2} \beta_{1} \sigma_{2}-j_{2} \sigma_{2}-j_{2} \sigma_{1}-\sigma_{3} \gamma_{1}-\sigma_{3} \gamma_{2}-\sigma_{3} \sigma_{2} \sigma_{1}+\sigma_{2} \gamma_{1}+\sigma_{1} \gamma_{2} ;$

$p_{3}=\beta_{2} \beta_{1} \omega_{2}^{2} j_{1}+\beta_{2} \beta_{1} \gamma_{2}-j_{2} \omega_{2}^{2} j_{1}-j_{2} \gamma_{2}-j_{2} \sigma_{2} \sigma_{1}-j_{2} \omega_{1}^{2} j_{1}-j_{2} \gamma_{1}-\sigma_{3} \sigma_{1} \omega_{2}^{2} j_{1}-\sigma_{3} \sigma_{1} \gamma_{2}-\sigma_{3} \sigma_{2} \omega_{1}^{2} j_{1}-\sigma_{3} \sigma_{2} \gamma_{1}+$ $\omega_{2}^{2} j_{1}^{2} \omega_{1}^{2}+\omega_{2}^{2} j_{1} \gamma_{1}+\gamma_{2} \omega_{1}^{2} j_{1}$

$p_{4}=-\sigma_{3} \omega_{2}^{2} j_{1}^{2} \omega_{1}^{2}+\left(-j_{2} \sigma_{1} \omega_{2}^{2}-\sigma_{3} \gamma_{2} \omega_{1}^{2}-\sigma_{3} \omega_{2}^{2} \gamma_{1}-j_{2} \sigma_{2} \omega_{1}^{2}\right) j_{1}-j_{2}\left(\sigma_{1} \gamma_{2}+\sigma_{2} \gamma_{1}\right)$

$p_{5}=-j_{2} j_{1}\left(\gamma_{2} \omega_{1}^{2}+j_{1} \omega_{2}^{2} \omega_{1}^{2}+\omega_{2}^{2} \gamma_{1}\right)$

The analytic determination of the eigenvalues solutions of Eq.(18) leads to quite large analytical expressions, this is why in order to find the parameter bands for which the system is stable, we recall the Routh- hurwitz stability criteria as follows;

All parameters of Eq.(18\}) must have the same sign, that is the positive sign since the first parameter is

positive. That is $p_{n}>0, n=0,1,2,3,4,5$. In addition,

$p_{2}-p_{0} p_{1}<0, p_{1} p_{4}-p_{2} p_{3}<0$ and $\frac{p_{2}}{p_{0}}\left(p_{2}-p_{0} p_{1}\right)-\frac{p_{0}}{p_{2}}\left(p_{1} p_{4}-p_{2} p_{3}\right)<0$

The numerical algorithm has been used to compute these eigenvalues, and the results are plotted in Figs.4(a,b), for $-1<X_{0}=Y_{0}<1$ and $-15<Z_{0}<15$, showing that the system admits some solutions with positive real part, meaning that the system model will admit unbounded or chaotic solutions.

(a)

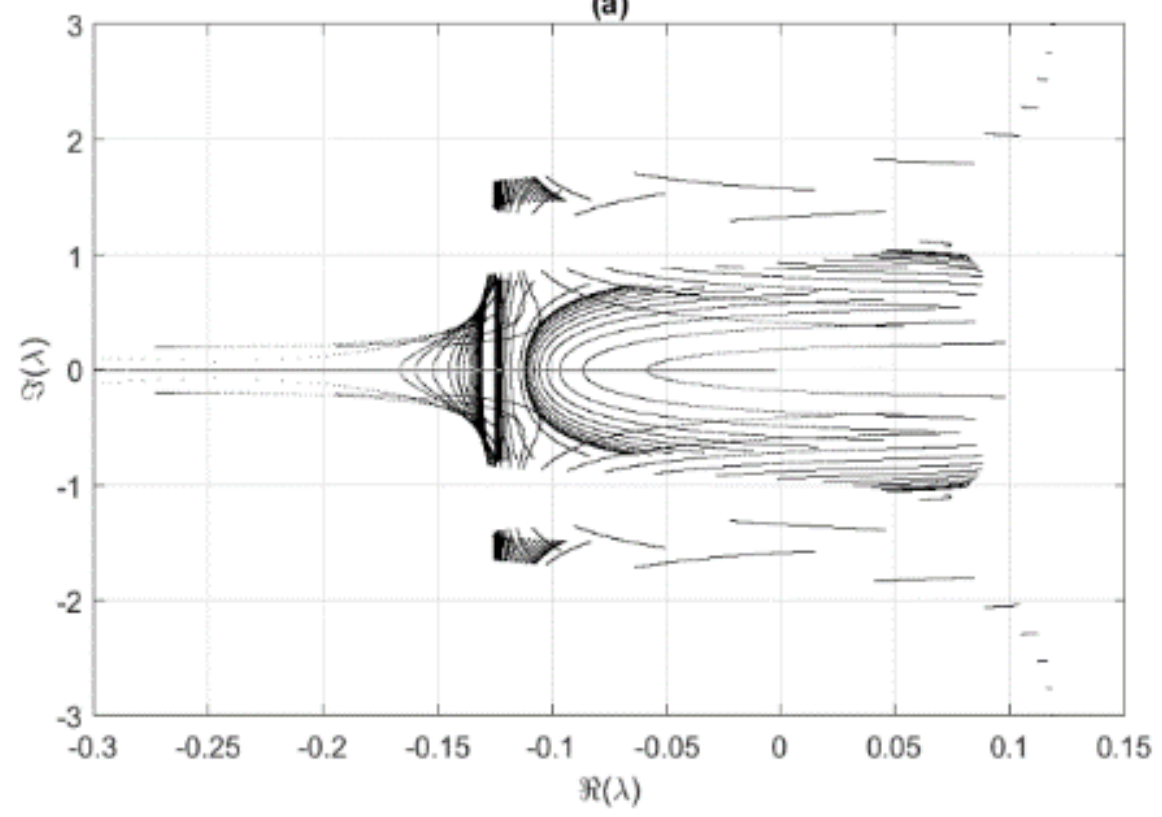




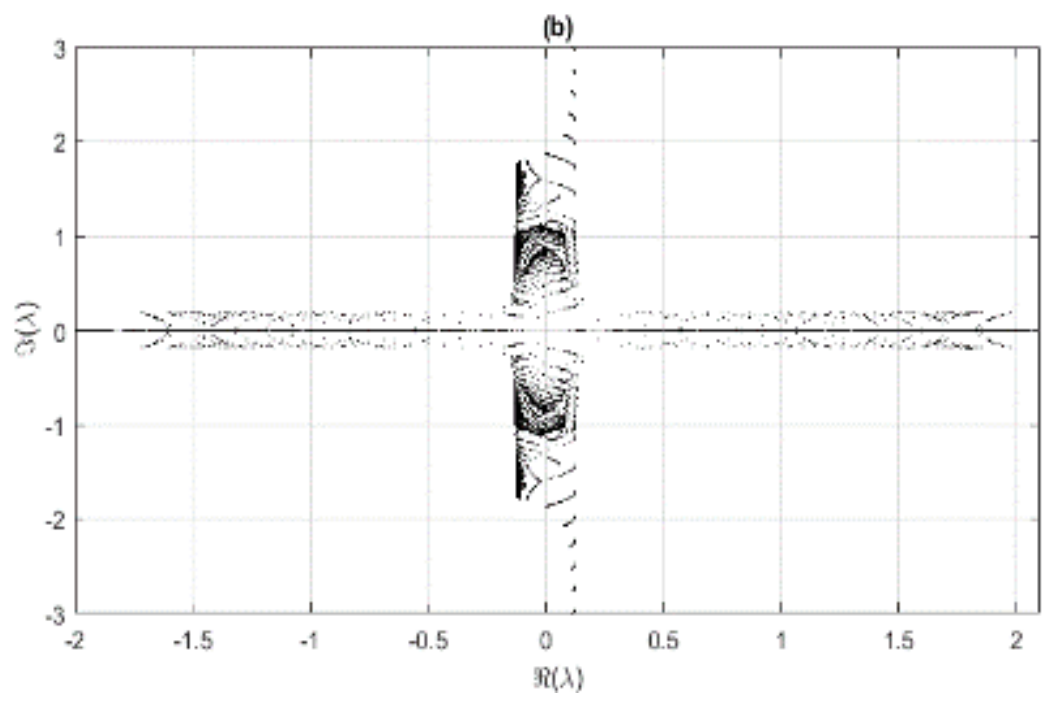

Figure 4 Real part versus imaginary part of solutions of Eq.(18), obtained for $\omega_{1}=\omega_{2}=\frac{1}{\sqrt{2}}, \boldsymbol{\beta}_{1}=\boldsymbol{\beta}_{2}=\mathbf{0 . 5}, \sigma_{1}=\sigma_{2}=$ 0. 25, $\sigma_{3}=0.25 \gamma_{1}=\gamma_{2}=1, \alpha_{1}=3, \alpha_{2}=1$, obtained by varying $-1<X_{0}=Y_{0}<1$ and $-15<Z_{0}<15$. (a): $\theta=$ $\pi / 2-0.02 \$$, and (b): $\theta=0.02$.

As one can see, the equation admits some solution with positive real part, which is the precursor sign of the existence of unbounded or chaotic solutions. The equation will also admit solution with zero real part, the well known Hopf bifurcation.

\section{Analytical solution of the system equation}

Amongst all the analytic approaches for the non-linear oscillations, the multiple time scales method (MTSM) has been chosen because it is the most adapted to the study of dynamical systems around resonance frequencies. In this paper, the damping, the coupling, the external excitation and the non-linearity are considered as global first order perturbations. Therefore let us write $\sigma_{1}=\epsilon \sigma_{10}, \sigma_{2}=\epsilon \sigma_{20}, \sigma_{3}=\epsilon \backslash s i g m a_{30}$,

$\gamma_{1}=\epsilon \gamma_{10}, \gamma_{2}=\epsilon \gamma_{20}, \beta_{1}=\epsilon \beta_{10}, \beta_{2}=\epsilon \beta_{20}, \alpha_{1}=\epsilon \alpha_{10}, \alpha_{2}=\epsilon \alpha_{20}$ and $E_{0}=\epsilon E$. In order to simplify our studies and to approach the solutions with the best accuracy, we will consider first the large amplitude oscillations, and approximated by Eq.(9), and secondly the weak amplitude given by Eq.(11):

\subsection{Case of the large amplitude oscillations: Discontinuous perturbed case}

for this particular case, Eq.(9) can be rewritten as:

$$
\left\{\begin{array}{c}
\ddot{X}+\epsilon \sigma_{10} \dot{X}+2 \omega_{1}^{2} X-2 \omega_{1}^{2} F_{1}+\epsilon \gamma_{10}\left(X-\varepsilon_{1} Y\right)-\epsilon \beta_{10} \dot{Z}=0, \\
\ddot{Y}+\epsilon \sigma_{20} \dot{Y}+2 \omega_{2}^{2} Y-2 \omega_{2}^{2} F_{2}+\epsilon \gamma_{20}\left(\varepsilon_{1} Y-X\right)=0, \\
\ddot{Z}-\epsilon \sigma_{30}\left(1-\alpha_{0} \dot{Z}^{2}\right) \dot{Z}+Z-\epsilon \alpha_{10} Z^{3}+\epsilon \alpha_{20} Z^{5}+\epsilon \backslash \operatorname{beta}_{20} \dot{X}=\epsilon E_{0} \cos (\Omega t),
\end{array},\right.
$$

with

$F_{i}=\frac{1}{2}\left[\operatorname{csgn}\left(X_{i}+\cos (\theta)\right)+\operatorname{csgn}\left(X_{i}-\cos (\theta)\right)\right]=\left\{\begin{array}{c}-1 \text { if } X_{i}<-\cos (\theta) \\ 0 \text { if }-\cos (\theta)<X_{i}<\cos (\theta) \\ 1 \text { if } X_{i}>\cos (\theta)\end{array}\right.$

where $X_{1}=X$ and $X_{2}=Y$. Let us set

$X(t)=X_{0}\left(T_{0}, T_{1}\right)+\epsilon X_{1}\left(T_{0}, T_{1}\right)+0\left(\epsilon^{2}\right), Y(t)=Y_{0}\left(T_{0}, T_{1}\right)+\epsilon Y_{1}\left(T_{0}, T_{1}\right)+0\left(\epsilon^{2}\right)$, 
$Z(t)=Z_{0}\left(T_{0}, T_{1}\right)+\epsilon Z_{1}\left(T_{0}, T_{1}\right)+0\left(\epsilon^{2}\right)$

with $T_{0}=t, T_{1}=\epsilon t, \frac{d}{d t}=D_{0}+\epsilon D_{1}$, and $D_{i}=\frac{\partial}{\partial T_{i}}$. Substituting Eqs.(23) into the set of Eqs.(21) and equating coefficients of like powers of $\$ \backslash$ epsilon $\$$, one obtains the following set of ordinary differential equations:

Equations at order $\epsilon^{0}$, then we have :

$\left\{\begin{array}{c}D_{0}^{2} X_{0}+2 \omega_{1}^{2} X_{0}=2 \omega_{1}^{2} F_{1} \\ D_{0}^{2} Y_{0}+2 \omega_{2}^{2} Y_{0}=2 \omega_{2}^{2} F_{2}, \\ D_{0}^{2} Z_{0}+Z_{0}=0\end{array}\right.$

admitting as solution:

$X_{0}=F_{1}+A_{1}\left(T_{1}\right) \backslash \cos \left(\omega_{1} \sqrt{2} T_{0}\right)+B_{1}\left(T_{1}\right) \sin \left(\omega_{1} \sqrt{2} T_{0}\right)$,

$Y_{0}=F_{2}+A_{2}\left(T_{1}\right) \cos \left(\omega_{2} \sqrt{2} T_{0}\right)+B_{2}\left(T_{1}\right) \sin \left(\omega_{2} \sqrt{2} T_{0}\right)$

$Z_{0}=A_{3}\left(T_{1}\right) \cos \left(T_{0}\right)+B_{3}\left(T_{1}\right) \sin \left(T_{0}\right)$,

At the order $\epsilon^{1}$, one has:

$$
\begin{aligned}
& D_{0}^{2} X_{1}+2 \omega_{1}^{2} X_{1}=-2 D_{0} D_{1} X_{0}-\sigma_{10} D_{0} X_{0}-\gamma_{10}\left(X_{0}-\varepsilon_{1} Y_{0}\right)+\beta_{10} D_{0} Z_{0}, \\
& D_{0}^{2} Y_{1}+2 \omega_{1}^{2} Y_{1}=-2 D_{0} D_{1} Y_{0}-\sigma_{20} D_{0} Y_{0}-\gamma_{20}\left(\varepsilon_{1} Y_{0}-X_{0}\right), \\
& D_{0}^{2} Z_{1}+Z_{1}=-2 D_{0} D_{1} Z_{0}+\sigma_{30}\left(1-\alpha_{0}\left(D_{0} Z_{0}\right)^{2}\right) D_{0} Z_{0}+\alpha_{10} Z_{0}^{3}-\alpha_{20} Z_{0}^{5}-\beta_{20} D_{0} X_{0}+E_{0} \cos (\Omega t),
\end{aligned}
$$

leading by taking into account (25) to

$$
\begin{aligned}
& D_{0}^{2} X_{1}+2 \omega_{1}^{2} X_{1}=-\left(2 \omega_{1} \sqrt{2} D_{1} B_{1}+\gamma_{10} A_{1}+\sigma_{10} B_{1} \omega_{1} \sqrt{2}\right) \cos \left(\omega_{1} \sqrt{2} T_{0}\right)+\left(2 D_{1} A_{1} \omega_{1} \sqrt{2}+\sigma_{10} A_{1} \omega_{1} \sqrt{2}-\right. \\
& \gamma_{10} B_{1} \sin \left(\omega_{1} \sqrt{2} T_{0}\right)+\gamma_{10}\left(\epsilon_{1} F_{2}-F_{1}\right)+\gamma_{-} 10 \epsilon_{1}\left(A_{2} \cos \left(\omega_{2} \sqrt{2} T_{0}\right)+B_{2} \sin \left(\omega_{2} \sqrt{2} T_{0}\right)\right)+\beta_{10}\left(B_{3} \cos \left(T_{0}\right)-A_{3} \sin \left(T_{0}\right)\right), \\
& D_{0}^{2} Y_{1}+2 \omega_{2}^{2} Y_{1}=-\left(2 \omega_{2} \sqrt{2} D_{1} B_{2}+\gamma_{20} A_{2}+\sigma_{20} B_{2} \omega_{2} \sqrt{2}\right) \cos \left(\omega_{2} \sqrt{2} T_{0}\right)+\left(2 D_{1} A_{2} \omega_{2} \sqrt{2}+\sigma_{-} 20 A_{2} \omega_{2} \sqrt{2}-\right. \\
& \left.\gamma_{20} B_{2}\right) \sin \left(\omega_{2} \sqrt{2} T_{0}\right)+\gamma_{20}\left(F_{1}-\epsilon_{1} F_{2}\right)+\gamma_{20}\left(A_{1} \cos \left(\omega_{1} \sqrt{2} T_{0}\right)+B_{1} \sin \left(\omega_{1} \sqrt{2} T_{0}\right)\right),
\end{aligned}
$$

$D_{0}^{2} Z_{1}+Z_{1}=\left[2 D_{1} A_{3}-\sigma_{30} A_{3}-\frac{5}{8} B_{3} R_{3}^{4} \alpha_{20}+\frac{3}{4}\left(\alpha_{10} B_{3}+\sigma_{30} \alpha_{0} A_{3}\right) R_{3}^{2}\right] \sin \left(T_{0}\right)+\beta_{20} \omega_{1} \sqrt{2}\left(A_{1} \sin \left(\omega_{1} \sqrt{2} T_{0}\right)-\right.$ $\left.\cos \left(\omega_{1} \sqrt{2} T_{0}\right) B_{1}\right)+E_{0} \cos (\Omega t)-\left[2 D_{1} B_{3}-\sigma_{30} B_{3}+\frac{5}{8} A_{3} R_{3}^{4} \alpha_{20}+\frac{3}{4}\left(-\alpha_{10} A_{3}+\alpha_{0} B_{3} \sigma_{30}\right) R_{3}^{2}\right] \cos \left(T_{0}\right)+\frac{1}{4}\left[\sigma_{30} B_{3}\left(3 A_{3}^{2}-\right.\right.$ $\left.\left.B_{3}^{2}\right) \alpha_{0}-\frac{1}{4} A_{3}\left(A_{3}^{2}-3 B_{3}^{2}\right)\left(5 \alpha_{20} R_{3}^{2}-4 \alpha_{10}\right)\right] \cos \left(3 T_{0}\right)-\frac{1}{4}\left[\sigma_{30} A_{3}\left(A_{3}^{2}-3 B_{3}^{2}\right) \alpha_{0}+\frac{1}{4} B_{3}\left(3 A_{3}^{2}-B_{3}^{2}\right)\left(5 \alpha_{20} R_{3}^{2}-\right.\right.$ $\left.\left.4 \alpha_{10}\right)\right] \sin \left(3 T_{0}\right)-\frac{1}{16} B_{3} \alpha_{20}\left(5 A_{3}^{4}-10 B_{3}^{2} A_{3}^{2}+B_{3}^{4}\right) \sin \left(5 T_{0}\right)-\frac{1}{16} A_{3} \alpha_{20}\left(5 B_{3}^{4}-10 B_{3}^{2} A_{3}^{2}+A_{3}^{4}\right) \cos \left(5 T_{0}\right)$

with $R_{3}^{2}=A_{3}^{2}+B_{3}^{2}$. The unknown amplitudes $A_{i}, B_{i}, i=1,2,3$ may now be determined by eliminating secular terms in Eqs.(27). However, because of the high dimensionality of the system, it is impossible to obtain unambiguously a set of secular equations valid for all frequencies. This is why it is necessary to find the solution near the unique possible resonance $\sqrt{2} \omega_{1}=\sqrt{2} \omega_{2}=\Omega=\omega_{e}$, with $\omega_{e}=1$. Let us then set

$\sqrt{2} \omega_{1}=1+\epsilon \xi_{1}, \sqrt{2} \omega_{2}=1+\epsilon \xi_{2}, \Omega_{1}=1+\epsilon \xi$

where $\xi_{1}, \xi_{2}$ and $\xi$ are are the tuning parameters expressing the quantitative nearness of the resonance frequencies $\sqrt{2} \omega_{1}, \sqrt{2} \omega_{2}$ and $\Omega$ with the fixed electric natural frequency $\omega_{e}=1$. Then, accounting Eqs.(28) into Eq.(31) leads to the following solvability conditions:

$2 \sqrt{2} \omega_{1} R_{1} D_{1} \phi_{1}+\gamma_{10} R_{1}-\gamma_{10} \epsilon_{1} R_{2} \cos \left(\psi_{1}\right)-\beta_{10} R_{3} \sin \left(\psi_{2}\right)=0$, 
$2 \sqrt{2} \omega_{1} D_{1} R_{1}+\sigma_{10} R_{1} \omega_{1} \sqrt{2}+\gamma_{10} \epsilon_{1} R_{2} \sin \left(\psi_{1}\right)-\beta_{10} R_{3} \cos \left(\psi_{2}\right)=0$,

$2 \sqrt{2} \omega_{2} D_{1} \phi_{2} R_{2}-\gamma_{20} R_{1} \cos \left(\psi_{1}\right)+\gamma_{20} \epsilon_{1} R_{2}=0$,

$2 \sqrt{2} \omega_{2} D_{1} R_{2}+\sigma_{20} R_{2} \omega_{2} \sqrt{2}-\gamma_{20} R_{1} \sin \left(\psi_{1}\right)=0$,

$2 R_{3} D_{1} \phi_{3}-\beta_{20} R_{1} \omega_{1} \sqrt{2} \sin \left(\psi_{2}\right)-\frac{3}{4} \alpha_{10} R_{3}^{3}+\frac{3}{8} \alpha_{20} R_{3}^{5}-E_{0} \cos \left(\psi_{3}\right)=0$,

$2 D_{1} R_{3}+\frac{3}{4} \sigma_{30} R_{3}^{3} \alpha_{0}+\beta_{20} R_{1} \omega_{1} \sqrt{2} \cos \left(\psi_{2}\right)-\sigma_{30} R_{3}-E_{0} \sin \left(\psi_{-} 3=0\right.$,

with

$\psi_{1}=\phi_{2}-\phi_{1}+\left(\xi_{1}-\xi_{2}\right) T_{1}, \psi_{2}=\xi_{1} T_{1}+\phi_{3}-\phi_{1}, \psi_{3}=\phi_{3}+\xi T_{1}$

These solvability conditions have a physical meaning if all phases are time independent, that is $D_{1} \psi_{1}=D_{1} \psi_{2}=D_{1} \psi_{3}=$ 0 , leading to $D_{1} \phi_{3}=-\xi, D_{1} \phi_{1}=\xi_{1}-\xi$ and $D_{1} \phi_{2}=\xi_{2}-\xi$. Otherwise $D_{1} R_{1}=D_{1} R_{2}=D_{1} R_{3}=0$. By solving the above set of equations, one has

$$
\begin{aligned}
& \psi_{1}=\arctan \left(\frac{2 \sigma_{20} \omega_{2}}{K_{1}}\right), \psi_{2}=\arctan \left(\frac{K_{2}}{2 K_{3}}\right), R_{1}=\frac{\beta_{10}\left(K_{1}^{2}+4 \sigma_{20}^{2} \omega_{2}^{2}\right) \sqrt{2}}{\sqrt{K_{2}^{2}+4 K_{3}^{2}}} R_{3} \\
& R_{2}=\frac{\gamma_{20} \sqrt{2}}{\sqrt{K_{1}^{2}+4 \sigma_{20}^{2} \omega_{2}^{2}}} R_{1}, K_{1}=4 \omega_{2}\left(\xi_{2}-\xi\right)+\gamma_{20} \varepsilon_{1} \sqrt{2}, \psi_{3}=-\arctan \left(\frac{3 \sigma_{30} R_{3}^{3} \alpha_{0}+8 K_{3} v-4 \sigma_{30} R_{3}}{4 K_{2} R_{3} v+8 \xi R_{3}+3 \alpha_{10} R_{3}^{3}-5 \alpha_{20} R_{3}^{5} / 2}\right) \\
& K_{2}=4\left(K_{1}^{2}+4 \sigma_{20}^{2} \omega_{2}^{2}\right)\left(\xi_{1}-\xi\right) \omega_{1}+\sqrt{2}\left(4 \sigma_{20}^{2} \omega_{2}^{2}-\sqrt{2} \gamma_{20} \varepsilon_{1} K_{1}+K_{1}^{2}\right) \gamma_{10} \\
& K_{3}=\sigma_{10} \omega_{1} K_{1}^{2}+4 \sigma_{10} \omega_{1} \sigma_{20}^{2} \omega_{2}^{2}+2 \varepsilon_{1} \gamma_{10} \gamma_{20} \sigma_{20} \omega_{2}, \quad v=\frac{2 \beta_{20} \beta_{10}\left(K_{1}^{2}+4 \sigma_{20}^{2} \omega_{2}^{2}\right)}{K_{2}^{2}+4 K_{3}^{2}} \omega_{1}
\end{aligned}
$$

while $R_{3}$ is the solution of the following equation:

$\frac{25}{64} \alpha_{20}^{2} R_{3}^{10}-\frac{15}{16} \alpha_{10} \alpha_{20} R_{3}^{8}+\left[-\frac{5}{4}\left(K_{2} v+2 \xi\right) \alpha_{20}+\frac{9}{6}\left(\alpha_{10}^{2}+\sigma_{30}^{2} \alpha_{0}^{2}\right)\right] R_{3}^{6}+\frac{3}{2}\left(\left(K_{2} v+2 \xi\right) \alpha_{10}-\sigma_{30}^{2} \alpha_{0}\right) R_{3}^{4}+3 K_{3} \sigma_{30} \alpha_{0} v R_{3}^{3}+$ $\left(\left(K_{2} v+2 \xi\right)^{2}+\sigma_{30}^{2}\right) R_{3}^{2}-4 K_{3} \sigma_{30} v R_{3}+4 K_{3}^{2} v^{2}-E_{0}^{2}=0$

Taking into account the above constraints imposed by Eq.(29 the direct solution of Eq.(27) reads

$X_{1}=\frac{\gamma_{10}}{2 \omega_{1}^{2}}\left(\epsilon_{1} F_{2}-F_{1}\right), Y_{1}=-\frac{\gamma_{20}}{2 \omega_{2}^{2}\left(\epsilon_{1} F_{2}-F_{1}\right)}, \quad Z_{1}=\frac{1}{128} R_{3}^{3}\left[\left(5 \alpha_{20} R_{3}^{2}-4 \alpha_{10}\right) \cos \left(3\left(T_{0}-\phi_{3}\right)\right)+4 \sigma_{30} \alpha_{0} \sin 3\left(T_{0}-\phi_{3}\right)\right]+$ $\frac{1}{384} R_{3}^{5} \alpha_{20} \cos \left(5\left(T_{0}-\phi_{3}\right)\right)$,

leading to the following solution:

$$
\begin{aligned}
& X(t)=\left(1-\frac{\gamma_{1}}{2 \omega_{1}^{2}}\right) F_{1}+\frac{\gamma_{1}}{2 \omega_{1}^{2}} \epsilon_{1} F_{2}+\frac{\beta_{1}\left(K_{1}^{2}+4 \sigma_{20}^{2} \omega_{2}^{2}\right) \sqrt{2}}{\sqrt{K_{2}^{2}+4 K_{3}^{2}}} R_{3} \cos \left(\Omega t+\psi_{2}-\psi_{3}\right) \\
& Y(t)=\left(1-\frac{\epsilon_{1} \gamma_{1}}{2 \omega_{2}^{2}}\right) F_{2}+\frac{\gamma_{1}}{2 \omega_{2}^{2}} F_{1}+2 \beta_{1} \gamma_{1} \sqrt{\frac{K_{1}^{2}+4 \sigma_{2}^{2} \omega_{2}^{2}}{K_{2}^{2}+4 K_{3}^{2}}} R_{3} \cos \left(\Omega t+\psi_{2 r}-\psi_{3}\right), \\
& Z(t)=R_{3} \cos \left(\Omega t-\psi_{3}\right)+\frac{1}{384} R_{3}^{5} \alpha_{2} \cos \left(5\left(\Omega t-\psi_{3}\right)\right)+\frac{1}{128} R_{3}^{3}\left[\left(5 \alpha_{2} R_{3}^{2}-4 \alpha_{1}\right) \cos \left(3\left(\Omega t-\psi_{3}\right)\right)+4 \sigma_{3} \alpha_{0} \sin (3(\Omega t-\right. \\
& \left.\left.\left.\psi_{3}\right)\right)\right],
\end{aligned}
$$

with now

$\psi_{2 r}=\arctan \left(\frac{1}{2} \frac{\left(\widetilde{K_{2}} \widetilde{K_{1}}-4 \omega_{2} \sigma_{2} \widetilde{K_{3}}\right)}{\widetilde{K_{1}} \widetilde{K_{3}}+\widetilde{K_{2}} \omega_{2} \sigma_{2}}\right), \psi_{2}=\arctan \left(\frac{\widetilde{K_{2}}}{2 \widetilde{K_{3}}}\right), \quad \psi_{3}=-\arctan \left(\frac{3 \sigma_{3} R_{3}^{3} \alpha_{0}+8 \widetilde{K_{3}} v-4 \sigma_{3} R_{3}}{4 \widetilde{K_{2}} R_{3} v+8(\Omega-1) R_{3}+3 \alpha_{1} R_{3}^{3}-5 \alpha_{2} R_{3}^{5} / 2}\right)$ 


$$
\begin{aligned}
\widetilde{K_{2}} & =4\left({\widetilde{K_{1}}}^{2}+4 \sigma_{2}^{2} \omega_{2}^{2}\right)\left(\omega_{1} \sqrt{2}-\Omega\right) \omega_{1}+\sqrt{2}\left(4 \sigma_{2}^{2} \omega_{2}^{2}-\sqrt{2} \gamma_{2} \varepsilon_{1} \widetilde{K_{1}}+{\widetilde{K_{1}}}^{2}\right) \gamma_{1}, \\
\widetilde{K_{3}} & =\sigma_{1} \omega_{1}^{2} \widetilde{K_{1}}+4 \sigma_{1} \omega_{1} \sigma_{2}^{2} \omega_{2}^{2}+2 \gamma_{1} \varepsilon_{1} \gamma_{2} \sigma_{2} \omega_{2}, v=\frac{2 \beta_{2} \beta_{1}\left({\widetilde{K_{1}}}^{2}+4 \sigma_{2}^{2} \omega_{2}^{2}\right)}{{\widetilde{K_{2}}}^{2}+4{\widetilde{K_{3}}}^{2}} \omega_{1}, \\
\widetilde{K_{1}} & =4 \omega_{2}\left(\omega_{2} \sqrt{2}-\Omega\right)+\gamma_{2} \varepsilon_{1} \sqrt{2} .
\end{aligned}
$$

(a)

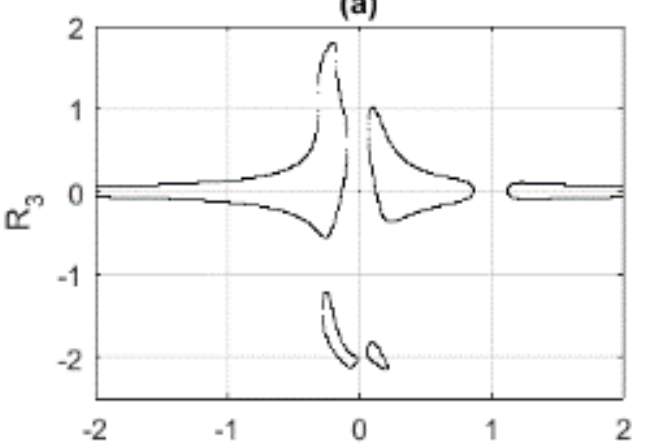

(c)

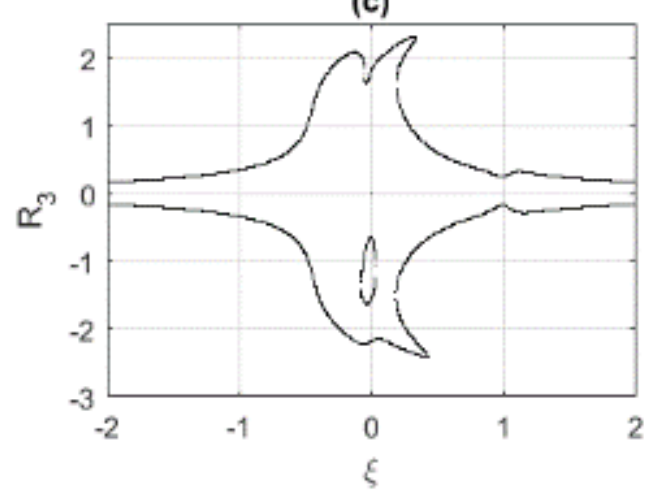

(b)

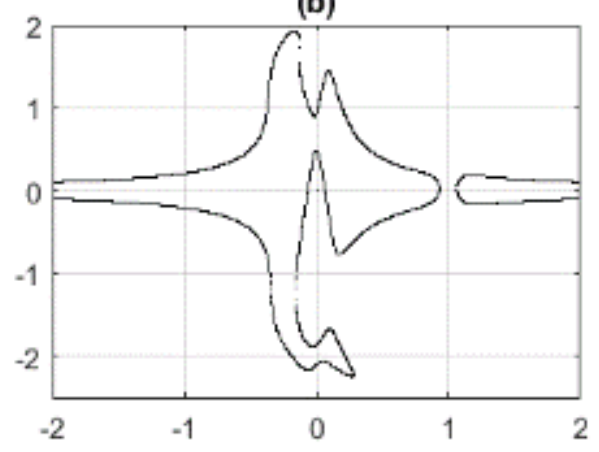

(d)

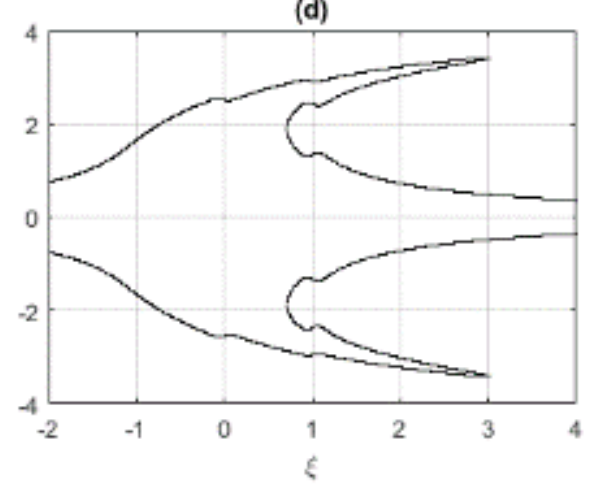

Figure 5 Bifurcation of the solution of Eq.(32), obtained for $\omega_{1}=\omega_{2}=\frac{1}{\sqrt{2}}, \beta_{10}=\beta_{20}=0.5, \sigma_{10}=\sigma_{20}=\sigma_{30}=$ $0.25, \gamma_{10}=\gamma_{20}=1, \alpha_{1}=0.3, \alpha_{2}=0.1, \xi_{1}=\xi_{2}=0, \varepsilon_{1}=1$ and for $(a): E_{0}=0.25,(b): E_{0}=0.4,(c): E_{0}=$ $0.65,(d): E_{0}=2.9$.

\subsection{Case of the weak amplitude oscillations: smooth case}

In order to study this case, let us take $\theta_{1}$ and $\theta_{2}$ such weak as $\theta_{i}=\epsilon^{1 / 2} \theta_{i 0}$, meaning that $\sin ^{2}\left(\theta_{i}\right) \approx \epsilon \theta_{i 0}^{2}$, and $\cos ^{2}\left(\theta_{i}\right) \approx$ $1-\epsilon \backslash$ thet $a_{i 0}^{2}$ leading Eq.(11) to

$$
\left\{\begin{array}{c}
\ddot{X}+\epsilon \sigma_{10} \dot{X}+2 \omega_{1}^{2} X-2 \epsilon \omega_{1}^{2} \theta_{1}^{2}\left(X+2 X^{3}+3 X^{5}\right)+\epsilon \gamma_{10}\left(X-\varepsilon_{1} Y\right)-\epsilon \beta_{10} \dot{Z}=0, \\
\ddot{Y}+\epsilon \sigma_{20} \dot{Y}+2 \omega_{2}^{2} Y-2 \epsilon \omega_{2}^{2} \theta_{2}^{2}\left(Y+2 Y^{3}+3 Y^{5}\right)+\epsilon \gamma_{20}\left(\varepsilon_{1} Y-X\right)=0, \\
\ddot{Z}-\epsilon \sigma_{30}\left(1-\alpha_{0} \dot{Z}^{2}\right) \dot{Z}+Z-\epsilon \alpha_{10} Z^{3}+\epsilon \alpha_{20} Z^{5}+\epsilon \beta_{20} \dot{X}=\epsilon \cos (\Omega t) .
\end{array}\right.
$$

By finding $X, Y$ and $\mathrm{Z}$ as outlined in Eq.(23), one obtains at order $\epsilon^{0}$ the same equation as in (24), but with $F_{1}=F_{2}=0$, admitting as solution:

$$
\begin{aligned}
& X_{0}=A_{1}\left(T_{1}\right) \cos \left(\omega_{1} \sqrt{2} T_{0}\right)+B_{1}\left(T_{1}\right) \sin \left(\omega_{1} \sqrt{2} T_{0}\right), Y_{0}=A_{2}\left(T_{1}\right) \cos \left(\omega_{2} \sqrt{2} T_{0}\right)+B_{2}\left(T_{1}\right) \sin \left(\omega_{2} \sqrt{2} T_{0}\right), \\
& Z_{0}=A_{3}\left(T_{1}\right) \cos \left(T_{0}\right)+B_{3}\left(T_{1}\right) \sin \left(T_{0}\right),
\end{aligned}
$$


(a)

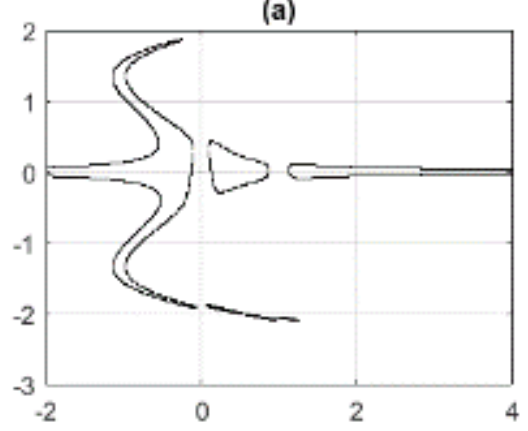

(c)

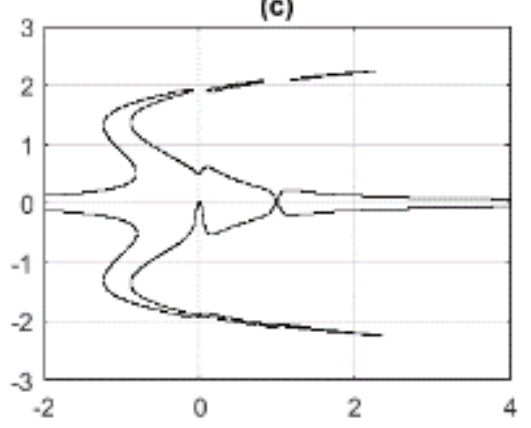

(b)

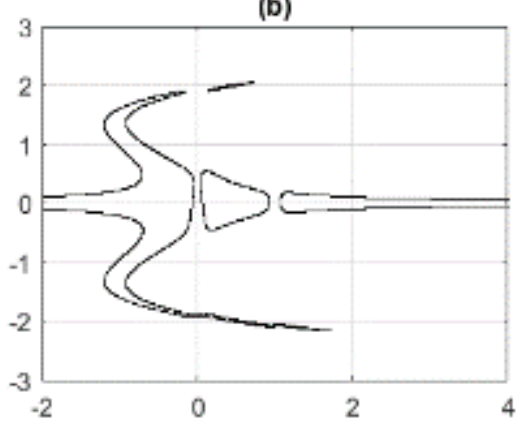

(d)

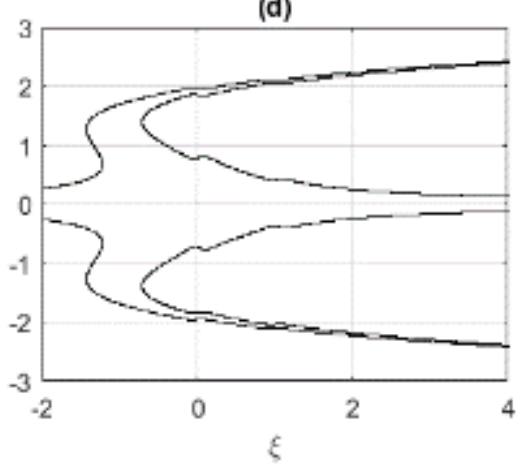

Figure 6 Bifurcation of the solution of Eq.(32), obtained for the same parameters as in Fig. 5, but with $\alpha_{1}=3$, and $\alpha_{2}=1$, obtained for $(a): E_{0}=0.25,(b): E_{0}=0.4,(c): E_{0}=0.65,(d): E_{0}=2.9$. while at order $\epsilon^{1}$ one has:

$$
\begin{aligned}
& D_{0}^{2} X_{1}+2 \omega_{1}^{2} X_{1}=-2 D_{0} D_{1} X_{0}-\sigma_{10} D_{0} X_{0}-\gamma_{10}\left(X_{0}-\varepsilon_{1} Y_{0}\right)+\beta_{10} D_{0} Z_{0}+2 \omega_{1}^{2} \theta_{1}^{2}\left(X_{0}+2 X_{0}^{3}+3 X_{0}^{5}\right) \\
& D_{0}^{2} Y_{1}+2 \omega_{1}^{2} Y_{1}=-2 D_{0} D_{1} Y_{0}-\sigma_{20} D_{0} Y_{0}-\gamma_{20}\left(\varepsilon_{1} Y_{0}-X_{0}\right)+2 \omega_{2}^{2} \theta_{2}^{2}\left(Y_{0}+2 Y_{0}^{3}+3 Y_{0}^{5}\right) \\
& D_{0}^{2} Z_{1}+Z_{1}=-2 D_{0} D_{1} Z_{0}+\sigma_{30}\left(1-\alpha_{0}\left(D_{0} Z_{0}\right)^{2}\right) D_{0} Z_{0}+\alpha_{10} Z_{0}^{3}-\alpha_{20} Z_{0}^{5}-\beta_{20} D_{0} X_{0} \\
& +E_{0} \cos (\Omega t)
\end{aligned}
$$

from where by proceeding as above, one has the following secularity conditions:

$$
\begin{aligned}
& 2 \omega_{1} \sqrt{2} D_{1} R_{1}-\beta_{1} R_{3} \cos \left(\psi_{2}\right)+\sigma_{1} R_{1} \omega_{-} 1 \sqrt{2}+\gamma_{1} \epsilon_{1} R_{2} \sin \left(\psi_{1}\right)=0, \\
& 2 R_{1} \omega_{1} \sqrt{2} D_{1} \phi_{1}-\frac{15}{4} \omega_{1}^{2} \theta_{1}^{2} R_{1}^{5}-\gamma_{1} \varepsilon_{1} R_{2} \cos \left(\psi_{1}\right)-3 \omega_{1}^{2} \theta_{1}^{2} R_{1}^{3}-2 \omega_{1}^{2} \theta_{1}^{2} R_{1}+\gamma_{1} R_{1}-\beta_{1} R_{3} \sin \left(\psi_{2}\right)=0, \\
& 2 \omega_{2} \sqrt{2} D_{1} R_{2}+\sigma_{2} R_{2} \omega_{2} \sqrt{2}-\gamma_{2} R_{1} \sin \left(\psi_{1}\right)=0, \\
& 2 R_{2} \omega_{2} \sqrt{2} D_{1} \phi_{2}-\frac{15}{4} \omega_{2}^{2} \theta_{2}^{2} R_{2}^{5}-\gamma_{2} R_{1} \cos \left(\psi_{1}\right)-3 \omega_{2}^{2} \theta_{2}^{2} R_{2}^{3}-2 \omega_{2}^{2} \theta_{2}^{2} R_{2}+\varepsilon_{1} \gamma_{2} R_{2}=0, \\
& 2 D_{1} R_{3}+\frac{3}{4} \sigma_{30} R_{3}^{3} \alpha_{0}+\beta_{20} R_{1} \omega_{1} \sqrt{2} \cos \left(\psi_{2}\right)-\sigma_{30} R_{3}-E_{0} \sin \left(\psi_{3}\right)=0 \\
& 2 R_{3} D_{1} \phi_{3}-\beta_{20} R_{1} \omega_{1} \sqrt{2} \sin \left(\psi_{2}\right)-\frac{3}{4} \alpha_{10} R_{3}^{3}+\frac{5}{8} \alpha_{20} R_{3}^{5}-E_{0} \cos \left(\psi_{3}\right)=0 .
\end{aligned}
$$



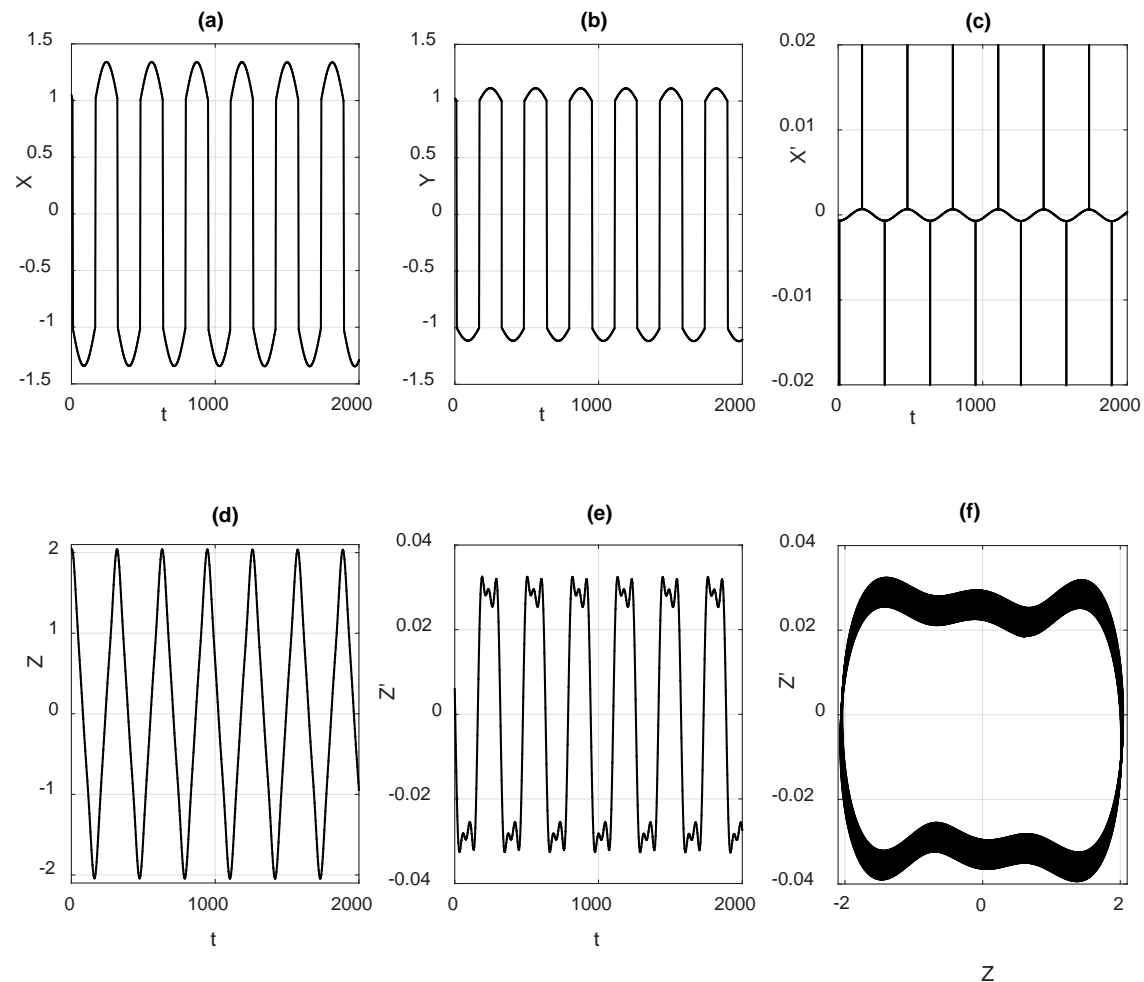

Figure 7 Profile of the solution given by Eq.(34), with parameters chosen as in Fig.6, with $\boldsymbol{\theta}_{\mathbf{1}}=\boldsymbol{\theta}_{\mathbf{2}}=\mathbf{0} . \mathbf{0 2}, \boldsymbol{E}_{\mathbf{0}}=$ $0.25, R_{3}=1.8$, and $\Omega=0.02$. As one can see the obtained solution is the bursting one.

$\psi_{1}, \psi_{2}$ and $\psi_{3}$ being defined as in Eq.(30). The even lines lead to the following expressions for $\psi_{i}, i=1,2,3$ :

$\psi_{1}=\arcsin \left(\frac{\sigma_{2} R_{2} \omega_{2}}{\gamma_{2} R_{1}} \sqrt{2}\right), \psi_{2}=\arccos \left(\frac{\sigma_{1} R_{1}^{2} \omega_{1} \gamma_{2}+\gamma_{1} \varepsilon_{1} R_{2}^{2} \sigma_{2} \omega_{2}}{\beta_{1} R_{3} \gamma_{2} R_{1}} \sqrt{2}\right)$,

$\psi_{3}=\arcsin \left(\frac{3 \sigma_{3} R_{3}^{4} \alpha_{0} \beta_{1} \gamma_{2}+8 \beta_{2} \omega_{1}^{2} \sigma_{1} R_{1}^{2} \gamma_{2}+8 \beta_{2} \omega_{1} \gamma_{1} \varepsilon_{1} R_{2}^{2} \sigma_{2} \omega_{2}-4 \sigma_{3} R_{3}^{2} \beta_{1} \gamma_{2}}{4 E_{0} \beta_{1} R_{3} \gamma_{2}}\right)$,

while the odd lines lead to:

$R_{1}=\frac{R_{2}}{\gamma_{2}} \sqrt{K_{2}^{2}+2 \sigma_{2}^{2} \omega_{2}^{2}} ; K_{2}=\frac{3}{4} \omega_{2}^{2} \theta_{2}^{2} R_{2}^{2}\left(5 R_{2}^{2}+4\right)-\delta_{2}$

according to the $4^{\text {th }}$ line, with $\delta_{2}=2 \omega_{2} \sqrt{2}\left(\xi_{2}-\xi\right)+\gamma_{2} \varepsilon_{1}-2 \omega_{2}^{2} \theta_{2}^{2}$, while the $2^{\text {th }}$ line leads to

$R_{3}=R_{1} \frac{\sqrt{2 \Gamma_{1}^{2} \beta_{1}^{2}+\left(K_{1}-\frac{\gamma_{1} R_{2}^{2}}{\left.\gamma_{2} R_{1}^{2} \varepsilon_{1} K_{2}\right)^{2}}\right.}}{\beta_{1}} ; K_{1}=\frac{3}{4} \omega_{1}^{2} \theta_{1}^{2} R_{1}^{2}\left(5 R_{1}^{2}+4\right)-\delta_{1}, \Gamma_{1}=\frac{\sigma_{1} \omega_{1}}{\beta_{1}}+\frac{\gamma_{1} \varepsilon_{1} R_{2}^{2} \sigma_{2} \omega_{2}}{\beta_{1} \gamma_{2} R_{1}^{2}}$,

With $\delta_{1}=2 \omega_{1} \sqrt{2}\left(\xi_{1}-\xi\right)+\gamma_{1}-2 \omega_{2}^{2} \theta_{2}^{2}$, The $6^{\text {th }}$ line yields

$\left[\left(2 \xi+\frac{\beta_{2} \omega_{1}}{\beta_{1} R_{3}^{2}} \sqrt{2}\left(K_{1} R_{1}^{2}-\frac{\gamma_{1}}{\gamma_{2}} \varepsilon_{1} K_{2} R_{2}^{2}\right)+\frac{3}{4} \alpha_{1} R_{3}^{2}-\frac{5}{8} \alpha_{2} R_{3}^{4}\right)^{2}+\left(\left(\frac{3}{4} R_{3}^{2} \alpha_{0}-1\right) \sigma_{3}+\frac{2 \beta_{2} \omega_{1} \Gamma_{1} R_{1}^{2}}{R_{3}^{2}}\right)^{2}\right] R_{3}^{2}=E_{0}^{2}$.

This equation is numerically solved and plotted in Fig.8 showing that the form of the obtained graph is different to that obtained in Fig.5. Meaning that the inclination angle could considerably affect the system's dynamics. 


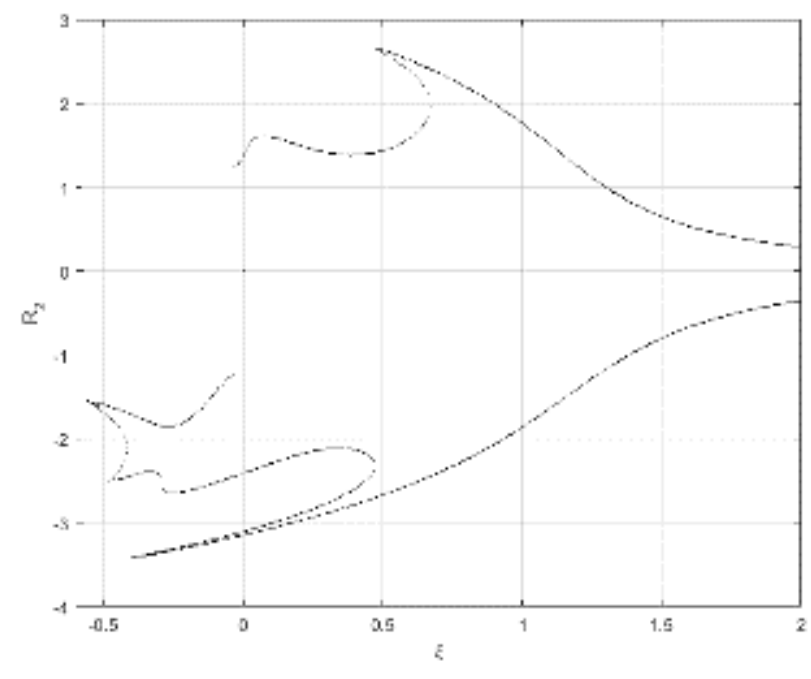

Figure 8 Bifurcation of the amplitude $\boldsymbol{R}_{\mathbf{2}}$ solution of Eq.(43) versus $\boldsymbol{\xi}$, and obtained for parameters chosen as in Fig.5, but with $\boldsymbol{E}_{\mathbf{0}}=\mathbf{0 . 5}$ and $\boldsymbol{\theta}=\mathbf{0 . 1}$.

\section{Numerical investigations}

\subsection{Preliminary}

In this section, we give numerical simulations results in order to confirm the theoretical results obtained in the previous sections and to find other new dynamics. The interesting problem is to analyze the parameter regions for which the system is regular or chaotic. The system having several parameters, each of them playing different and virtual roles on the system behavior, we will then limit our study by chosen both the driven frequency and inclination angles as control parameters. Otherwise the values of other parameters are fixed as follow:

$\omega_{1}=\omega_{2}=\frac{1}{\sqrt{2}}, \beta_{1}=\beta_{2}=0.5, \sigma_{1}=\sigma_{2}=\sigma_{3}=0.25, \gamma_{1}=\gamma_{2}=1, \alpha_{1}=3 \alpha_{2}=1, \varepsilon_{1}=1, E_{0}=0.25$.

Using then these parameters, the system Eq.(7) is numerically solved by using the fourth order Runge Kutta scheme, with the time step always kept $\omega_{e} \delta t=10^{-2}$.

\subsection{Effect of the driven frequency $\Omega$}

To begin, let us mention that there are some tools to measure, predict, and quantify chaotic dynamics in mechanical systems. The queries are how to recognize chaos and predict their occurrence? The answer is that they can be recognized by looking period doubling and homoclinic bifurcations in the systems. They can also be quantified by Lyapunov exponents [15]. While the maximum Lyapunov exponent is positive, the systems remains chaotic. In this subsection we analyze the effects of the driven frequency $\Omega$ by keeping constant the other parameters as given in Eq.(44) including the inclination angles which are $\theta_{1}=\theta_{2}=\pi / 4$. The first results in the such of case is plotted in Fig.(9), showing the regular behavior zones for $0.8<\Omega<0.8926$ and $0.94<\Omega<0.96$. Elsewhere we have the bifurcation bands, with some regular behaviors windows in the bifurcation band. As we can see Fig.(9), (a,b and c) are bifurcation diagrams for variables X,Y and Z, respectively which are in agreement with (d), that is the maximum Lyapunov exponent. In order to qualify the signals generated, we have plotted in Figs.(10,11 and 12), the signal profiles at the left column, the phase portraits at the middle, and the frequency spectrum at the right. In all these figures, the top lines are for variable X, the middles for Y and the bottoms for Z. Figs.(10 and 11) are obtained for $\Omega=0.02$ and $\Omega=0.5$, which belong to chaotic zones. The signals generated for $\mathrm{X}$ and $\mathrm{Y}$ are chaotic impulses, while that generated for $\mathrm{Z}$ are chaotic bursting. It is obvious that the bursting or impulses widths are more larger for $\Omega=0.02$ than $\Omega=0.5$. As one can see from the last columns of these figures, the spectrum frequency of these figures have two noisily bands, justifying then that the system is chaotic. Figure(12), obtained for $\Omega=0.85$ chosen in the regular band frequency shows that X and Y are regular impulses signals, while $\mathrm{Z}$ is the regular bursting, even thought the bursting width for $\mathrm{Z}$ is too weak, but the phase portrait of $(12)\left(c_{2}\right)$ justifies our conclusion. The spectrum frequency of these figures presents some regular picks. 

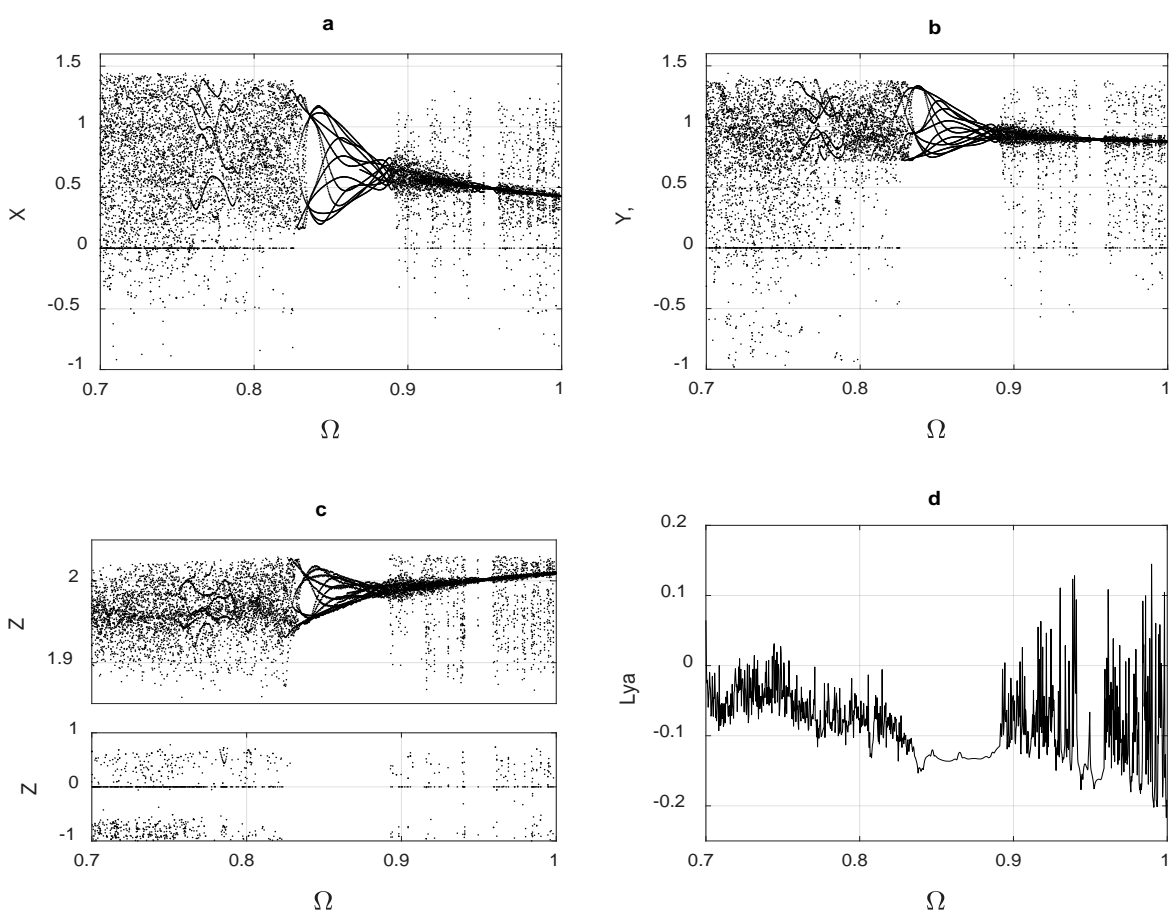

Figure 9 Bifurcation diagram obtained for parameters given in Eq.(44), for $\boldsymbol{\alpha}_{\mathbf{1}}=\mathbf{3} \boldsymbol{a n d} \boldsymbol{\theta}=\boldsymbol{\pi} / \mathbf{4}$.
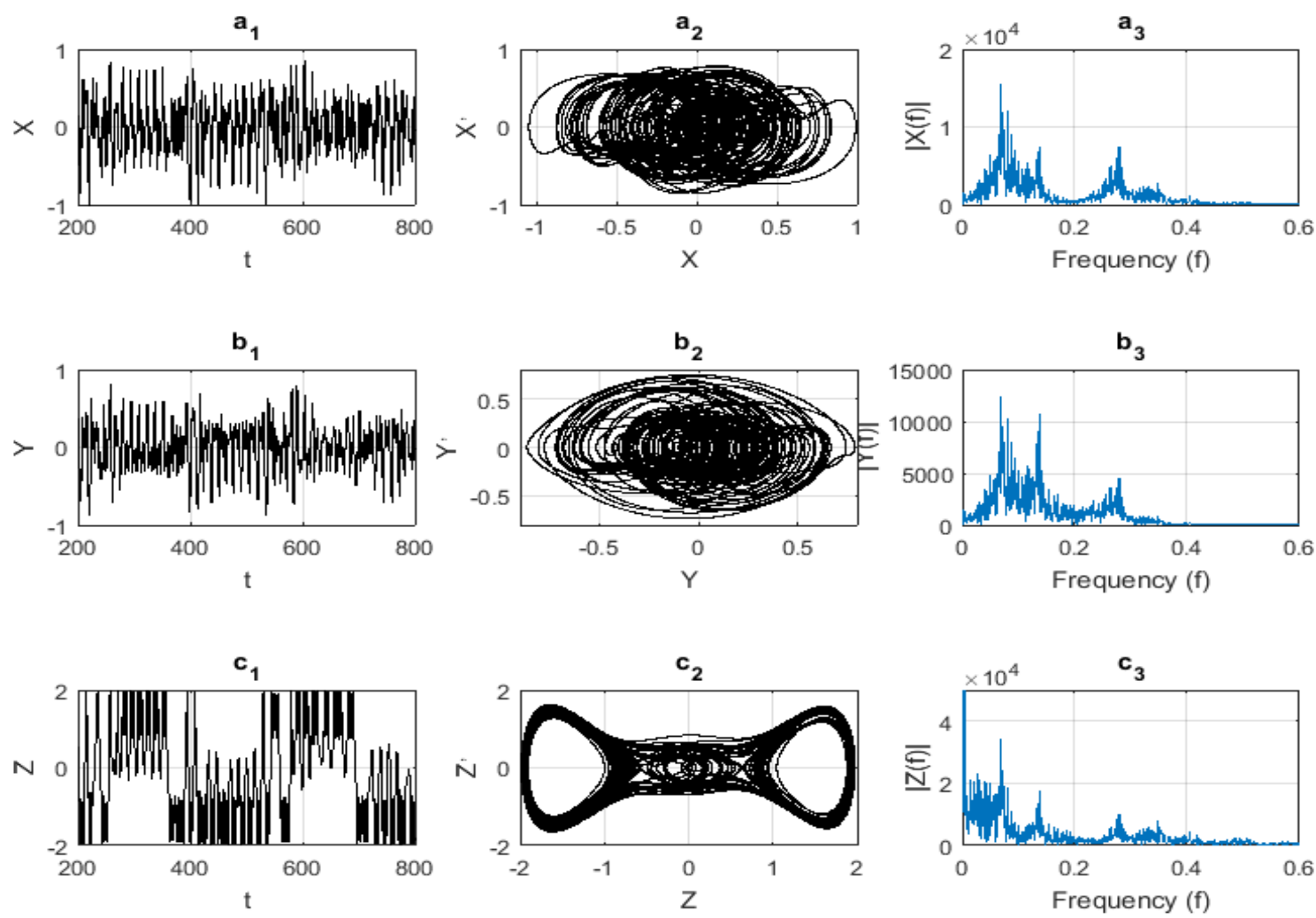

Figure 10 Left: Solution, Middle: phase portrait, and right: frequency spectrum, obtained for parameters chosen as in Fig.( 9), and for $\Omega=0: 02$ 

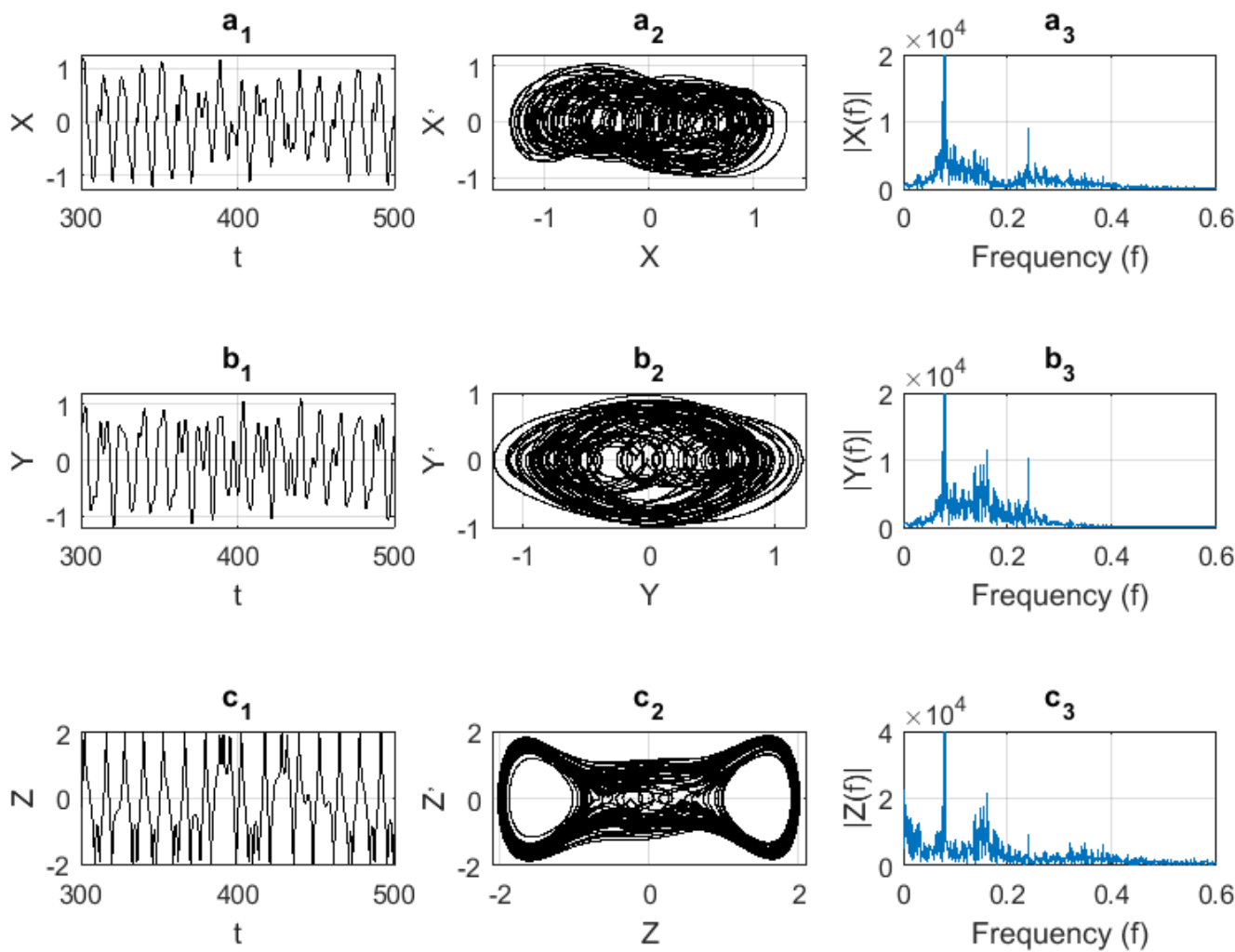

Figure 11 Left: Solution, Middle: phase portrait, and right: frequency spectrum, obtained for parameters chosen as in Fig.( 9), and for $\Omega=0: 5$.
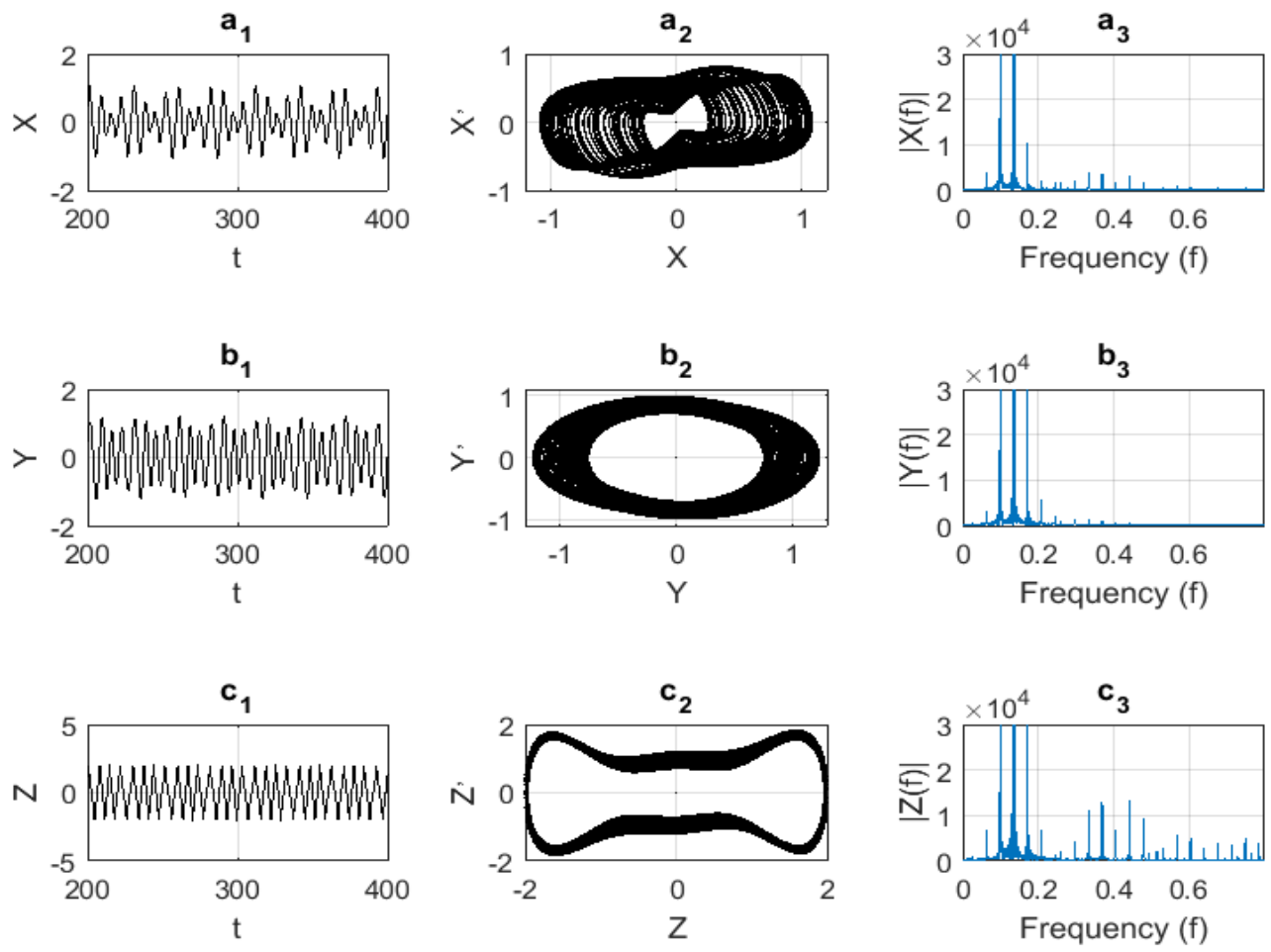

Figure 12 Left: Solution, Middle: phase portrait, and right: frequency spectrum, obtained for parameters chosen as in Fig.( 9), and for $\Omega=0: 85$ 


\subsection{Effect of the inclination angles $\theta_{1}=\theta_{2}=\theta$}

Let us analyze in this sub-section the effects of the inclination angles $\theta_{1}=\theta_{2}=\theta$, by keeping constant the other parameters as given in Eq(44). In Figs.(13) and (14), we have the bifurcation diagrams for $\Omega=0.02$, that is for the weak frequency value, and $\Omega=0.85$, that is for the large frequency value.

In Fig.(13) obtained for weak value of $\Omega$, the regular band is found for the inclination angle $0 \leq \theta<0.239$, that is for the less inclination. Otherwise for $\theta>0.239$, chaos is found, with the appearing of regular behavior windows.

However for large value of $\Omega$ as shown in Fig.(14), the regular behavior is found for $\theta>0.62$, that is when springs are more inclined. Figure (15) shows the regular train of kink signal obtained for $\theta_{1}=\theta_{2}=\theta=0.4$ belonging to chaotic band of Fig.(13), agreeing the belonging of regular frequency windows inside the chaotic bands. In Fig.(16) we have the periodic signals generations for $\theta_{1}=\theta_{2}=\theta=1.2$ belonging to regular windows.
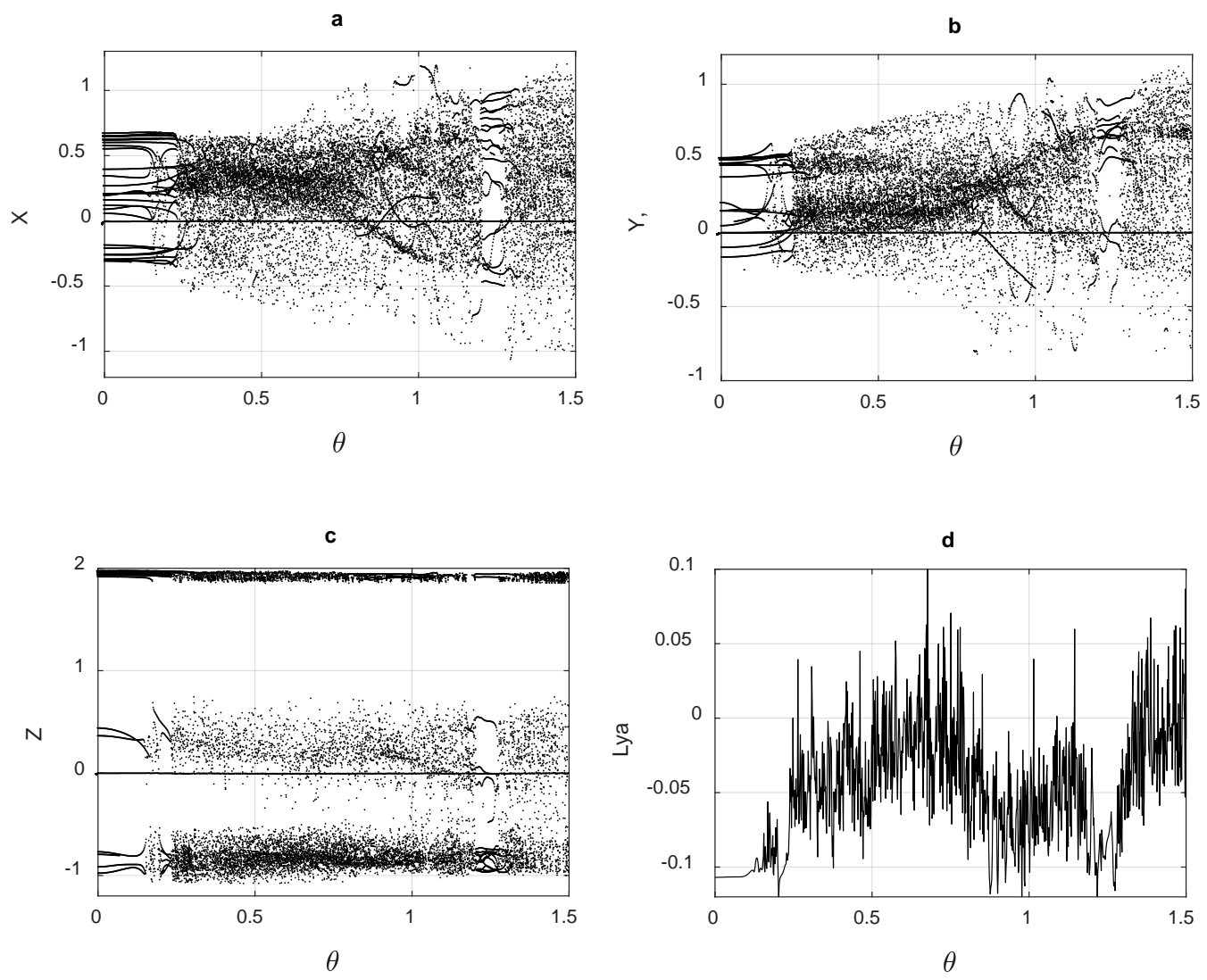

Figure 13 Bifurcation diagram for parameters chosen as in Fig.(14) and for $\Omega=0: 02$.

\section{Discussion}

From the above results, it is obvious that:

The system is very sensitive for the varying values of the driven frequency $\Omega$ and the inclination angles $\theta_{1}$ and $\theta_{2}$. The system is also sensitive to nonlinearity coefficients and the amplitude of the driven signal, but this last case is not investigated in the present work.

The system can generate bursting-like signals for very low frequencies, which are regular or chaotic according to the inclination angles.

The system can also generate regular or chaotic periodic kinks for higher frequency, according also to the inclination angles. 

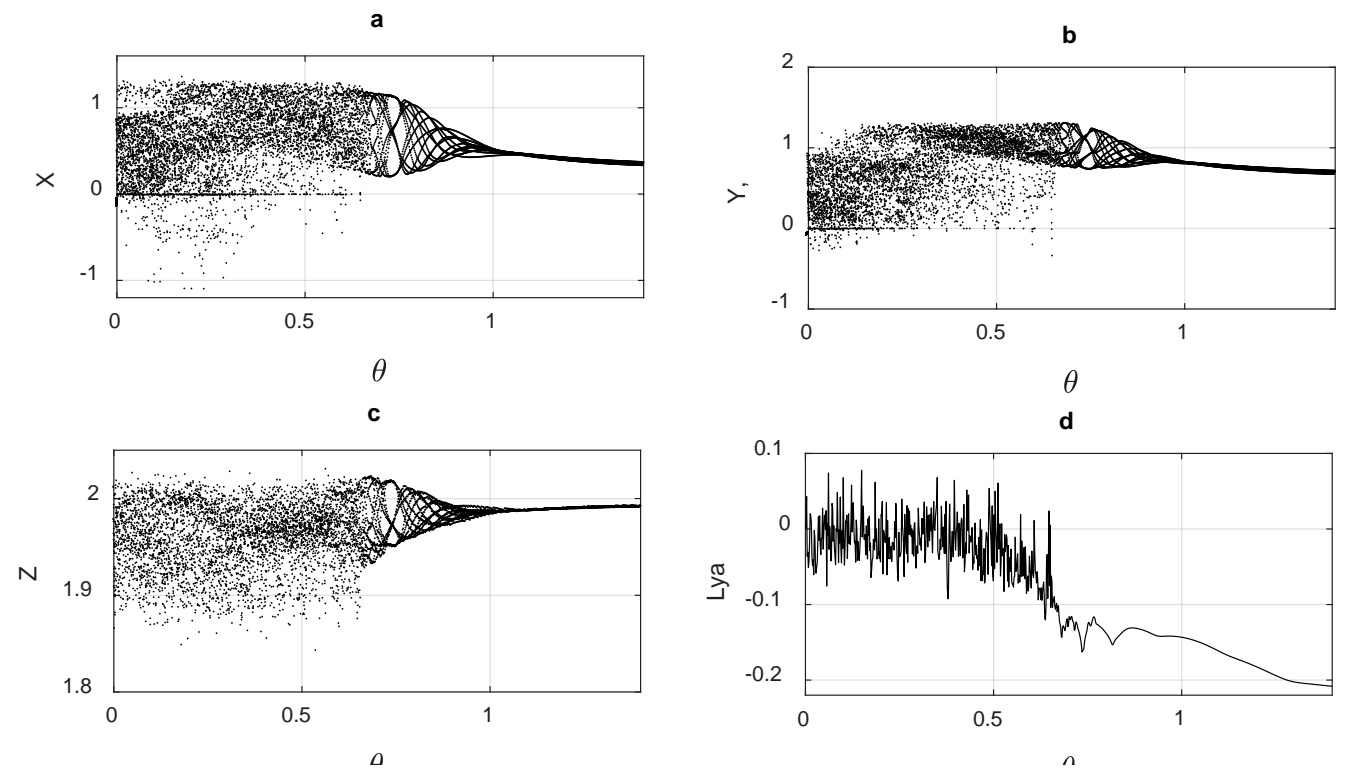

Figure 14 (a,b,c): Bifurcations diagrams for variables $X$; $Y$ and $Z$, respectively, while (d) is the maximum Lyapunov exponent. The parameters are taken as giving in Eq.(44) and for $\alpha 1=3$, and $\Omega=0: 85$.
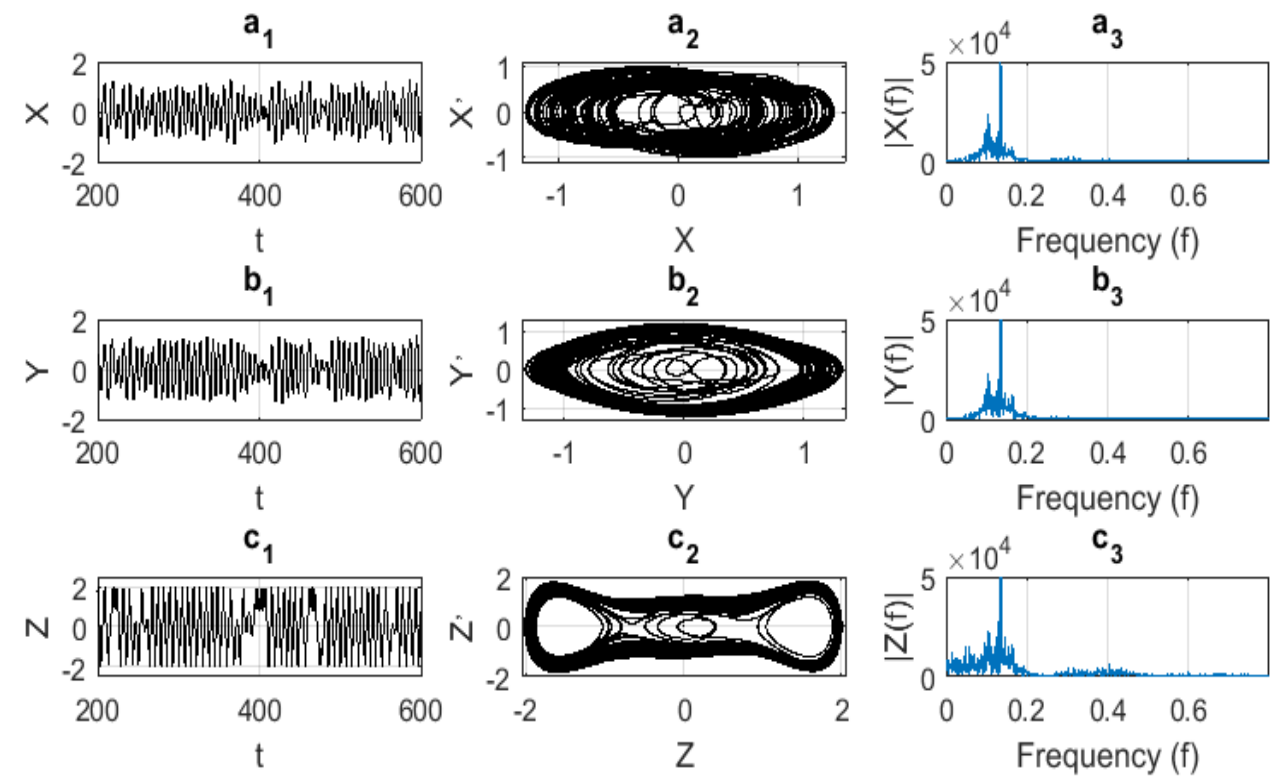

Figure 15 Left: Solution, Middle: phase portrait, and right: frequency spectrum, obtained for parameters chosen as in Fig.( 14), and for $\theta_{1}=\theta_{2}=\theta=0: 4$. 

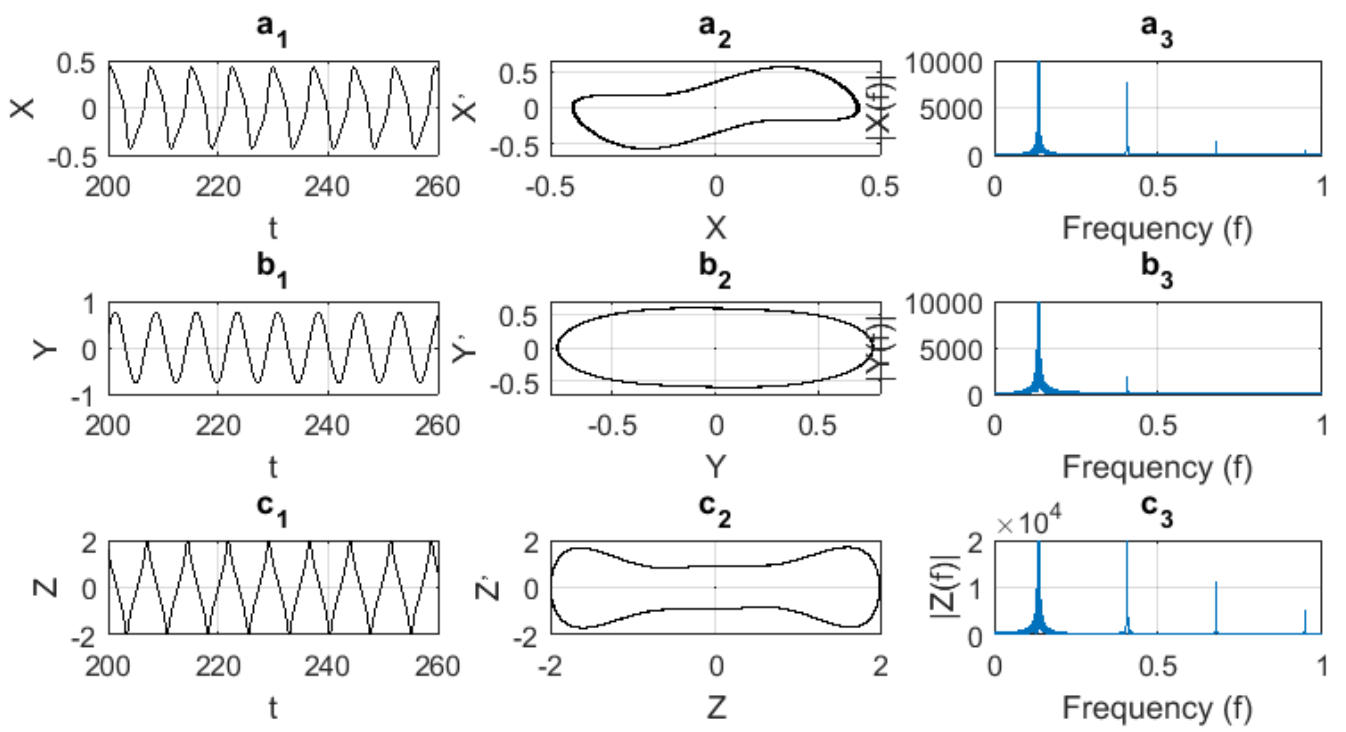

Figure 16 Left: Solution, Middle: phase portrait, and right: frequency spectrum, obtained for parameters chosen as in Fig.(14), and for $\theta_{1}=\theta_{2}=\theta=1: 2$.

\section{Conclusion}

In this paper, we have investigated the dynamics of the nonlinear electromechanical device, consisting of the mechanical part with two outputs and the electrical part both couplet magnetically. The mechanical part consisted of two nonlinear elastically coupled oscillators with strong irrational nonlinearities having smooth or discontinuous characteristics, where nonlinearity was due to the geometric configuration, while the electrical part is the $\phi^{6}$ - Rayleigh equation. By using the Lagrangian formulation, the model equations have been established and used to investigate the equilibrium points and their stabilities. Next by using the multiple time scales method, the analytical solutions have been found both for the case of large amplitude, and the weak amplitude, respectively leading to an interesting bifurcation sets of the equilibria for varying values of control parameters, that are the inclination angles and driven frequency. Next through numerical investigations, the bifurcation diagrams, the maximum Lyapunov exponents, the frequency spectrums and phase portraits have been plotted to demonstrate the complex transitions of the system to chaos. By plotting the output signals for the electrical and mechanical parts, it appeared that chaotic impulses, chaotic bursting and the train of kink signals were generated by the system, according to the tuning parameters.

From the above obvious results, it appeared that the use of the coupled oscillator constructed in the present work can represent an excellent way to multi-signal generations, allowing their possible applications as hammer, saw or sieve, with two outputs. However, our worry is what would be the effect of the single-impact or vibro-impact energy on the system dynamics, is it possible to insert in the system a supplementary component able to control chaos by annihilating them. The works is this light will constitute our future investigations.

\section{Compliance with ethical standards}

\section{Acknowledgments}

Adoum Danao Adile would like to express his gratitude to "Centre national d'Appui à la recherche(CNAR)",

Chad, for the financial support of the present research.

\section{Disclosure of conflict of interest}

All authors would like to declare that there is no conflict of interest relevant to this article. 


\section{References}

[1] MV Tchakui and P Woafo, Dynamics of three unidirectionally coupled autonomous Duffing oscillators and application to inchwprm piezoelectric motors: Effects of the coupling coefficient and delay, Chaos 2016; 26: 113108

[2] H Simo and J K Dutt; Effects of depth potential on the dynamics of Duffing system and chaos control: numerical and electronic implementation, India J.Phys. 2020; 94:361-370

[3] Fabien Kenmogne, David Yemélé and Paul Woafo, "Electrical dark compacton generator: Theory and simulations", Phys. Rev. E 85, 056606.2012

[4] HAN YanWei, CAO QingJie1, CHEN YuShu1 and WIERCIGROCH Marian, "A novel smooth and discontinuous oscillator with strong irrational nonlinearities", Sci China-Phys Mech Astron October 2012; Vol. 55 No. 10

[5] Doupac M, Beale D G, Overfelt R, "A. Three-dimensional lumpedmass/lumped spring modeling and nonlinear behaviour of a levitated droplet", Nonlin Dyn, 2005: 42:2542

[6] Boubaker B B, Haussy B Mesoscopic fabric models using a discrete massspring approach: Yarn-yarn interactions analysis. J Mater Sci, 2005; 40:59255932

[7] Terumichi $Y$, Ohtsuka M, Yoshizava M, et al. Nonstationary vibrations of a string with time-varying length and a mass-spring system attached at the lower end. Nonlin Dyn, 1997; 12:3955

[8] U H Hegazy, 'Dynamics and control of a self-sustained electromechanical seismographs with time-varying stiffness', Meccanica 2009; 44:355368

[9] Y Chembo Kouomou, P Woafo, 'Triple resonant states and chaos control in an electrostatic transducer with two outputs', Journal of Sound and Vibration2004; 270:7592

[10] Herve, Simo and Paul Woafo; Bursting oscillations in electromechanical systems, Mechanics Research Communications 2011; 38:537541

[11] C A Kitio Kwuimy, B Nana and P Woafo; Experimental bifurcations and chaos in a modified self-sustained macro electromechnaical system; Journal of Sound and Vibration 2010; 329:31373148

[12] Field S, Kaus M, Moore M G Chaotic system of falling disc. Nature, 1997; 388:252254

[13] Lenci S, Rega G Regular nonlinear dynamics and bifurcations of an impacting system under general periodic excitation. Nonlin Dyn, 2003; 34:249268

[14] Perter L V, Gabor D. Symmetry, optima and bifurcation is structure design. Nonlin Dyn, 2006; 43:4758

[15] M Lakshmanan and S Rajaseekar, Nonlinear Dynamics: Integrability, Chaos and Patterns, Springer, 2003. 